\title{
Mediengesellschaft und Medialisierung
}

\author{
Kurt Imhof
}

In den Auseinandersetzungen um Gesellschaftsbegriffe in der Sozialtheorie sowie in der Medialisierungsforschung kristallisieren sich im Wechselspiel von theoretischen Entwicklungen und empirischen Einsichten Kerne einer kommunikationswissenschaftlichen Theoriebildung heraus, die die gegenstandsbezogene Orientierung der Kommunikationswissenschaft an den Massenmedien öffnet. Dabei spielen der Mediengesellschaftswie der Medialisierungsbegriff eine wichtige Rolle, während die sozialtheoretische Debatte über Gesellschaftsbegriffe diesen Erkenntnisanspruch begründen und orientieren kann. Es zeigt sich, dass den Erkenntnismitteln der Kommunikationswissenschaft wichtige Aufgaben in der sozialwissenschaftlichen Analyse der Gesellschaft zuwachsen. Dieser Beitrag zeichnet diesen Kristallisationsprozess nach und erarbeitet einen Vorschlag für die Fassung des Mediengesellschafts- wie des Medialisierungsbegriffs.

Schlagwörter: Mediengesellschaft, Medialisierung, Öffentlichkeit, Differenzierung

Der Beitrag" beginnt mit der Darlegung der jüngeren sozialtheoretischen Auseinandersetzungen um eine adäquate Konzeptualisierung von ,Gesellschaft ${ }^{\top}$ und zeigt, dass sich die insbesondere seit den 1980er Jahren stattfindende, systemfunktionalistische Engführung der Sozialtheorie auf die funktionale Differenzierung genauso ihrem Ende zuneigt wie die Verdrängung der anforderungsreichen Kategorie ,Öffentlichkeit' aus der sozialwissenschaftlichen Theoriebildung. In der breiten Diskussion über neue Gesellschaftsbegriffe gewinnen die stratifikatorische und segmentäre Differenzierung, die Interdependenzen aller drei Differenzierungsformen und damit die klassische Dreifaltigkeit der Differenzierungstheorie genauso wie die Öffentlichkeit wieder an Bedeutung. Dadurch kommt gleichzeitig auch die Dialektik von Kultur und Struktur wieder zu ihrem Recht. In dieser Entwicklung erhöht sich das Differenzierungsniveau der Sozialtheorie insgesamt, und den Tendenzen ihrer entweder strukturalistischen oder kulturalistischen Engführung erwächst Widerstand. Dadurch können neben der Differenzierung auch wieder die Bedingungen der Integration der modernen Gesellschaft thematisiert werden (1. Funktionale, segmentäre und stratifikatorische Differenzierung und Integration).

Vor diesem Hintergrund von Differenzierung und Integration wird dann der ,neue Strukturwandel der Öffentlichkeit in Gestalt der Ausdifferenzierung des Mediensystems in seinen wichtigsten Elementen diskutiert. Dabei gilt es, den Auswirkungen dieses Prozesses auf die öffentliche Kommunikation und seinen Effekten auf andere Hand-

Für diesen Beitrag haben viele Diskussionen im und um das Mediensymposium Luzern und am IPMZ - Institut für Publizistik- und Medienwissenschaft der Universität Zürich beigetragen. Allen, die sich geduldig mit dem Autor auseinander setzten, sei herzlich gedankt. Ebensolcher Dank richtet sich an die anonymen Gutachter und die Redaktionskommission, die eine erste Version dieses Aufsatzes validiert haben. Den Reaktionen eines Teils der Gutacher auf den ursprünglich wesentlich leidenschaftlicher formulierten Text war zu entnehmen, dass der Versuch, aus sozialtheoretischer Perspektive einen Gesamtbezug zur Kommunikationswissenschaft zu machen, Lücken zu benennen und dies auf die vereinseitigte Dominanz der funktionalen Differenzierung zurückzuführen, ein diffiziles Unternehmen darstellt. 
lungssysteme (Medialisierung) differenzierungstheoretisch möglichst unverkürzt Rechnung zu tragen (2. Neuer Strukturwandel der Öffentlichkeit).

Damit sind die Grundlagen geschaffen, um einen differenzierungstheoretisch reflektierten Begriff der Mediengesellschaft zu entwickeln. Diese Heuristik eignet sich auch, um die Medialisierungseffekte in funktionaler, stratifikatorischer und segmentärer Dimension auf der Mikro-, der Meso- und der Makroebene zu erfassen und diejenigen Forschungsfelder zu benennen, in denen die Kommunikationswissenschaft noch zu wenig zum Verständnis der modernen Gesellschaft beiträgt (3. Fazit: Mediengesellschaft und Medialisierung). ${ }^{1}$

\section{Funktionale, segmentäre und stratifikatorische Differenzierung und Integration}

Die Geschichte der sozialtheoretischen Auseinandersetzung mit der Gesellschaft wie mit der Öffentlichkeit und der öffentlichen Meinung ist durch die Berücksichtigung der funktionalen, der stratifikatorischen und der segmentären Differenzierung gekennzeichnet. Diese differenzierungstheoretische Dreidimensionalität der Sozialtheorie rekurriert auf die Einsicht, dass die Gesellschaft vertikal, horizontal und funktional gegliedert ist und dass sich diese Differenzierungen wechselseitig beeinflussen. Die Klassiker der Sozialwissenschaften berücksichtigten in ihren Begriffsarchitekturen allesamt die funktionale, die stratifikatorische und die segmentäre Differenzierung und betrachteten die Interdependenzen dieser Differenzierungsformen immer mit Blick auf den sozialen Wandel. Durch die Beschäftigung mit der Differenzierung erkannten oder bestätigen sie ihre Entwicklungshypothesen ${ }^{2}$ und gewannen dadurch ihre Heuristiken für die Analyse des sozialen Wandels auf der Makro-, der Meso- und der Mikroebene, also in der Gesellschafts-, der Organisations- und Institutionen- wie der Handlungstheorie. Ihr Interesse galt mal eher der einen oder anderen Differenzierung, aber nie nur einer allein und nie nur in einer Richtung. Im Gegenteil: Differenzierungsdynamiken in der einen Dimension beobachteten sie mit Blick auf Differenzierungsdynamiken in der anderen Dimension und dies selbstverständlich auch mit Blick für Entdifferenzierungen. ${ }^{3}$

Und wie hielten sie es mit der Öffentlichkeit, der öffentlichen Meinung, der Ideologie, der öffentlichen Kommunikation? Gleichgültig, wohin wir blicken: Die Öffentlich-

1 Die Ausführungen konzentrieren sich auf die Auswirkungen der Mediengesellschaft auf die demokratische Selbststeuerung und damit vorab auf die politische Kommunikation und auf die Strukturen des Politischen. Damit werden hier insbesondere die Auswirkungen auf die Ökonomie vernachlässigt (vgl. hierzu Eisenegger 2005).

2 Organizistische Entwicklungskonzepte (Spencer), Widerspruch zwischen Produktivkräften und Produktionsverhältnissen (Marx), Rationalisierung (Weber, Horkheimer/Adorno), Individualisierung (Simmel), Symbolität des Sozialen und Reflexivität durch Kommunikation (Pragmatismus: Dewey, Mead); von der mechanischen zur organischen Solidarität (Durkheim); von der Gemeinschaft zur Gesellschaft (Tönnies); Modernisierung als Rationalisierung, funktionale Differenzierung, Universalisierung und Demokratisierung (Parsons).

3 Selbstverständlich affizieren sich diese Differenzierungstypen wechselseitig. Abschichtungen müssen im Rahmen der Ethnisierung oder der religiösen Aufladung sozialer Ungleichheit auch als segmentäre Differenzierungen beschrieben werden. Funktionale Differenzierungen können segmentäre, nationalstaatliche Differenzierungen schwächen, beispielsweise im Kontext der Globalisierung von Wirtschaft und Politik, und segmentäre Differenzierungen können in die gegebenen Strukturen funktionaler und stratifikatorischer Differenzierung ,einziehen', wenn die Gesellschaft durch Immigration ethnisch oder religiös unterschichtet und desintegriert wird. 
keit und die öffentliche Meinung ${ }^{4}$ bildet neben den darin beachteten Differenzierungen immer auch das Medium, dass die moderne Gesellschaft jenseits ihrer Differenzierung zusammenhält. Denn der Blick auf die Dynamik der Differenzierung zieht die Frage nach der Integration der Gesellschaft nach sich. Neben dem, was wir heute Systemintegration nennen können, im Sinne der funktionalen Verknüpfung von Handlungsfolgen und in unserer alltäglichen Konfrontation mit den funktional, segmentär und stratifikatorisch gegliederten Strukturen externalisierter Sozialität (Peters 1993b) setzten sie sich mit der Sozialintegration ${ }^{6}$ auseinander und entdeckten in mannigfachen Begriffssprachen das, was Marx mit dem Terminus der (falschen) Ideologie einholte, Weber Ideen, Charisma und Gemeinsamkeitsglauben nannte, was bei Durkheim als mechanische und organische Solidarität, bei Tönnies als Gemeinschaft, bei Simmel als soziale Kreise und bei Schütz als Lebenswelt erscheint. Dabei thematisierten die Klassiker die Öffentlichkeit als überwölbendes Moment von Sozialität und zwar gleichzeitig mit Blick für die funktionalen, stratifikatorischen und segmentären Partikularitäten, die sich in der öffentlichen Kommunikation oder in den (festen, flüssigen oder gasförmigen) öffentlichen Meinungen spiegeln. Denn sowohl Differenzierung als auch Integration manifestieren sich in der öffentlichen Kommunikation, die gleichzeitig Agens von Differenzierung und Integration ist. Über resonanzreiche Akteure kandidieren sozialstrukturelle Differenzierungen permanent um Aufmerksamkeit, verdrängen sich dabei gegenseitig und verwandeln sich durch öffentliche Kommunikation in politisch relevante Selbst- und Fremdbeschreibungen von Gruppen oder Teilsystemen. Freilich muss dieser Prozess immer in seiner Wechselwirksamkeit gelesen werden: Resonanzreiche Selbst- und Fremdbeschreibungen verdinglichen sich in Gestalt sozialstruktureller Differenzierungen. Das ist der eine wesentliche Grund, warum die Öffentlichkeit die Krux der Sozialtheorie ist. Sie bildet Differenzierung ab, kann sie zum Thema machen, treibt sie über wechselseitige Beobachtung und Konflikt an und überwölbt gleichzeitig die Differenzierungen nur schon durch die wechselseitige Beobachtung, die sie ermöglicht.

Der andere wesentliche Grund besteht in der Lösung des Problems einer friedlichen sozialen Ordnung, die die Aufklärungsbewegung den anciens régimes entgegenstellte. Die absolut zentrale Bedeutung, die die Aufklärung der Vernunft und Tugend schaffenden Öffentlichkeit in Abgrenzung zur Privatheit verlieh, kreierte die wichtigsten Basisnormen und -institutionen der modernen Gesellschaft, schuf also den Bauplan der Moderne und damit das, womit sich die Sozialtheorie und die Sozialforschung zu beschäftigen hat. In der Beschreibung und in der Konzeptualisierung der Differenzierung der modernen Gesellschaft mitsamt ihrer Öffentlichkeit gipfelt deshalb die Komplexität der Sozialtheorie, und ihre Geschichte lässt sich als immer wieder neue Bewältigung dieser Komplexität lesen (Imhof 2006 [1996]).

4 Als Kollektivsingular des Aufklärungsverständnisses bedeutet dies eine gemeinsame Überzeugung. Bei Tönnies (1922) ist das die stets groß geschriebene „Öffentliche Meinung“ und steht den stratifikatorisch und segmentär-herkunftsbezogenen „gemeinschaftlichen“ Gruppenpositionen, den „öffentlichen Meinungen“ gegenüber. Öffentliche Meinung im Sinne einer über Befragungstechniken eruierten Summe individueller Meinungen stand nicht im Fokus des sozialtheoretischen Interesses.

5 Formen delegierter Macht und Zuständigkeit (funktionale Handlungsbereiche, Institutionen, Hierarchien, Rollen, Verfahren).

6 Historisch vorgefundene, sozial produzierte Infrastrukturen; über Sozialisationsprozesse reproduzierte Symbolität alles Sozialen in Gestalt der kommunikativen Vermittlung lebensweltlichen Wissens. Die Unterscheidung von System- und Sozialintegration stammt von Lockwood (1964, 244-257). 
Trotzdem haben sich die Sozialwissenschaften und mit diesen auch die Kommunikationswissenschaft insbesondere von den 1960er bis in die 1990er Jahre kaum mehr mit der Öffentlichkeit beschäftigt und die Differenzierungstheorie immer mehr auf die funktionale Differenzierung reduziert. Hierfür gibt es eine Fülle von Ursachen, hervorgehoben werden die drei wichtigsten:

- Wesentlich ist der Aufstieg des systemtheoretischen Funktionalismus im Kontext der Modernisierungseuphorie der 1950er und 1960er Jahre in der Ära des Kalten Krieges und des sozialmarktwirtschaftlichen Gesellschaftsmodells. Die entwicklungsdeterministische Vernachlässigung der stratifikatorischen und segmentären Differenzierung (Wehler 1975, Giddens 1976, 325-345) in diesem Paradigma ist an sich schon bei der Geburt der Sozialwissenschaften bzw. schon in der durchaus utopischen Aufklärungsphilosophie angelegt, erreichte jedoch erst im Kalten Krieg im Rahmen der Modernisierungstheorien einen ersten Höhepunkt. Mit Rückgriff auf Weber, Marx, Durkheim und Pareto verknüpfte Talcott Parsons die Rationalisierung des Handelns und der sozialen Beziehungen, die Universalisierung der Normen und Werte, die Individualisierung und die funktionale Differenzierung der Gesellschaft in spezialisierte Teilsysteme zu einer Modernisierungsaxiomatik (Parsons 1976 [1951]). Klassen und Schichten und das damit verbundene politische Konfliktpotenzial wie segmentäre Aus- und Abschließungsprozesse erhielten die Konnotation des Vergangenen in der Gegenwart und verloren, weit über die Modernisierungstheorien hinaus, an Einfluss in der Gesellschaftsanalyse. ${ }^{7}$ Dafür wurde der Blick der Sozialwissenschaften für die funktionale Differenzierung geschärft: Gesellschaft wurde immer mehr durch die Heuristik funktionaler Teilsysteme hindurch beobachtet, und diese Heuristik verdinglichte sich so weit, dass sich die sozialwissenschaftlichen Fächer in die Gegenstandsbereiche aufteilten, in die der Funktionalismus die Welt gliederte. Gleichzeitig verlor die Kategorie Öffentlichkeit an Bedeutung, weil sie sich dieser Heuristik partout nicht fügt.

- Der zweite Grund liegt in der sowohl durch den Funktionalismus als auch durch die Renaissance der marxistischen Gesellschaftskonzepte in den späten 1960er und frühen 1970er Jahren beförderten, strukturalistischen Vereinseitigung der Sozialwissenschaften. Sie führte unter anderem zu einem Aufschwung der Sozialgeschichte, die sich scharf von der traditionalen ereignis- und personenorientierten Geistesgeschichte absetzte. Im Licht dieser „Basis-Überbau-Renaissance“ und befördert durch die Kulturindustriethesen von Horkheimer/Adorno (1969 [1944/1947]) wurde zwar seitens der neomarxistischen Konzepte die stratifikatorische Differenzierung betont, die Öffentlichkeit allerdings als bloße Verdoppelung der gesellschaftsstrukturellen Basis (,falsches Bewusstsein') einerseits und als Ausdruck einer vereinseitigten Rationalität der Moderne andererseits abgewertet. Öffentliche Kommunikation konnte in dieser Perspektive nur noch Objekt von Ideologiekritik und Niedergangsbeschrieben werden, und die kritische Theorie beschränkte sich auf den erkenntnis-

7 Diese überlistete sich mit der doppelten Interpretation ethnischer Vergemeinschaftung als Restbestand der Vormoderne in der Moderne und als irrationale Reaktion auf die Modernisierung selbst: Ethnizität diente genauso als Beweis der Modernisierungstheorie wie die Nicht-Existenz von ethnischen Spannungen. Dieser Reduktionismus muss zu „Anomalien“ oder „Irritationen“ führen, weil die Effekte der stratifikatorischen und der segmentären Entwicklungsdynamiken in Termini der funktionalen Differenzierung weder adäquat beobachtet noch erklärt werden können. Vgl. Esser 1988, 235-248; Nassehi 1990, 261-282; Imhof 1997, 861-875; Joas 1996, 13 27; Wimmer 1996, 173-198. 
theoretischen Pfad der Gesellschaftsanalyse (vgl. Negt/Kluge 1972; Habermas 1972). Beides, die strukturalistische Vereinseitigung von Funktionalismus und Neomarxismus und die ideologiekritische, erkenntnistheoretische Ergänzung des Strukturalismus durch die neomarxistischen Ansätze wie durch die Frankfurter Schule verunmöglichten eine Perspektive, in der die öffentliche Kommunikation als primäres Zugangsportal zur Analyse der modernen Gesellschaft - mitsamt ihren Differenzierungen und ihrer Dialektik von Struktur und Kultur - entdeckt werden konnte.

- Die dritte Ursache lässt sich dann umgekehrt auf die Verselbstständigung von Kultur („cultural turn“) und Kommunikation („linguistic turn“) in der poststrukturalistischen Gegenbewegung der 1980er Jahre zurückführen, die ihren Ausdruck in den Theorien der Postmoderne, in vielen Individualisierungstheorien und im radikalkonstruktivistischen Systemfunktionalismus fand. Der Postmodernismus führte zur Unterbelichtung der Sozialstruktur und zur Abwertung der Kategorie der Rationalität, dementierte entsprechend die Möglichkeit der intentionalen (demokratischen) Steuerung des Sozialen und entwickelte keinen Gesellschaftsbegriff. Gleichzeitig reduzierte die vor allem im deutschen Sprachraum in den 1980er und 1990er Jahren resonanzreiche radikalkonstruktivistische Systemtheorie die Differenzierungstheorie ausschließlich auf die denkbar essentialistischste Form der funktionalen Differenzierung in Gestalt der - den Naturwissenschaften entstammenden - Autopoiesisvorstellung und verneinte ebenfalls die Steuerungsfähigkeit der modernen Gesellschaft (Luhmann 1984, 242-285). Diese funktionale Differenzierung, die Durkheimsche „Arbeitsteilung ", lässt sich in ihrer Interessen- und Akteurlosigkeit am besten als das normfreie, "quasi-transzendental“ waltende Prinzip evolutionärer Entwicklung der sozialtheoretischen Arbeit voranstellen: Im Funktionalismus kulminiert deshalb der Positivismus der Sozialwissenschaften. Diese evolutionstheoretische Perspektive beförderte die Vernachlässigung der historischen Dimension sozialwissenschaftlicher Analyse und sie führte zum zweiten Höhepunkt der Abwertung der analytischen Kategorien der stratifikatorischen und segmentären Differenzierung und der damit verbundenen Reflexion auf materielle Ungleichheit, unterschiedliche Zugangschancen, Versäulungs- wie Marginalisierungseffekte und ihre sozialen Folgen. Für eine kurze Zeit schien die Sozialtheorie Gesellschaft anders als Patchwork eigenlogischer Teilsysteme nicht mehr wahrnehmen zu können, zumal die inzwischen für Teilsysteme zuständigen sozialwissenschaftlichen Fächer, ihr' Teilsystem eifersüchtig verteidig(t)en. Gemessen an der differenzierungstheoretischen ,Dreifaltigkeit' der Sozialwissenschaften ist diese Reduktion auf die funktionale Differenzierung eine vereinseitigende Perspektive auf die Gesellschaft. Trotz der von der Aufklärung ererbten Fortschrittsorientierung war den Klassikern klar, dass eine systematische Beobachtung der Gesellschaft ohne Berücksichtigung der stratifikatorischen und segmentären Differenzierungsdynamiken mehr als nur einen ,blinden Fleck' hat. Diese blinden Flecke wurden jedoch im Banne der weitgehend funktionalistisch argumentierenden Globalisierungstheorien zum einen, des Postmodernismus und des Individualisierungsparadigmas zum anderen, $\mathrm{zu}$ groß. Die Beschleunigung stratifikatorischer und segmentär-religiöser und -ethnischer Strukturwandelsprozesse erhielt mitsamt ihren mannigfachen sozialpolitischen Effekten zu wenig Beachtung. Zum politischen Credo des Steuer-, Standort- und Aufmerksamkeitswettbewerbs im ausgehenden Jahrtausend passten offensichtlich Sozialtheorien, die implizit oder explizit von einem „Ende der Geschichte“ ausgingen, die "Postmoderne“ oder die „zweite Moderne“ verkündeten, die Welt in strikt eigenlogische Teilsysteme aufteilten und/oder durch eine euphorisierte Fokussierung einer digitalen (Medien-Welt-)Kultur unterschiedliche Zugangschancen und partikuläre Öffent- 
lichkeitsbezüge wie auch segmentär und stratifikatorisch geprägte Öffentlichkeitsarenen übersahen.

Diese in Anbetracht der Pfadabhängigkeiten sozialwissenschaftlichen Denkens überraschend starke Anpassung weiter Teile der Sozialtheorie an den Zeitgeist musste über kurz oder lang in Aporien und zu einer Korrektur führen. Maßgeblich hierfür waren Einsichten in die Folgeeffekte des Steuer-, Standort- und Aufmerksamkeitswettbewerbs, in dem in stratifikatorischer und segmentärer Hinsicht unterschiedliche Opportunitätsspielräume auch unterschiedliche Chancen verteilen. Überspitzt formuliert begannen sich der Sozialtheorie Verwerfungen stratifikatorischen und segmentären Typs wieder von ihrem Gegenstand her aufzudrängen: Die zunehmende Prekarität der Arbeitsverhältnisse bis weit in den Mittelstand hinein und die politischen Effekte, die von der globalisierungskritischen Bewegung bis hin zu den Erfolgen nationalistischer oder sezessionistischer („Legas“) rechtsbürgerlicher Protestparteien in Westeuropa („Standortnationalismus") reichen, sowie die Entfesselung ethnischer und religiöser Spannungen bis hin zum Bürgerkrieg und zu „riots“ in den desintegrierten Vorstädten westeuropäischer Metropolen entziehen sich jedem funktionalistischen Reduktionismus. Überspitzt ist diese Darstellung allerdings deshalb, weil sich innerhalb der Sozialtheorie auch jene "Schulen“ erneuerten, die an der tradierten Problemkomplexität umfassender Differenzierung einerseits, der Integrationsfrage andererseits weiterarbeiteten (etwa: Giddens 1992, Bourdieu 1987, Joas 1992, Alexander 1982, Münch 1995), Sozialstruktur bzw. System und Lebenswelt wieder zu vereinen trachteten (Habermas 1981, 1992, Peters 1993a) oder im Kontext der „Cultural Studies“ über die Aneignung des Hegemoniebegriffs Gramscis, Foucaults Diskurstheorie und Bourdieus Struktur und Kultur integrierende Theorie sozialer Felder (neben der diskurs- und medienanalytischen Erforschung von Subkulturen) auch wieder zur dialektischen Analyse von Spannungspotenzialen in Kultur und Struktur finden (Hall 1996 [1980], 13-42, 1992; Winter 2001, 67-158). Nimmt man das zusammen, dann zeugen die außergewöhnlich intensiven Debatten über Gesellschaftsmodelle seit den 1990er Jahren (vgl. Pongs 1999/2000) von einem Unbehagen in Sozialtheorie und Sozialforschung angesichts der offensichtlichen analytischen Defizite zum einen und von einer Renaissance nicht reduktionistischen, sozialtheoretischen Denkens zum anderen.

Wenn man nun diese Orientierungsdebatten um adäquate Gesellschaftsbegriffe seit den 1990er Jahren zu reflektieren versucht, dann lässt sich entdecken, dass sich das Differenzierungsniveau wieder erhöht. In diesen Gesellschaftsbegriffsdebatten lassen sich drei Differenzierungsprozesse erkennen, denen gesteigerte Aufmerksamkeit zukommt. Es handelt sich dabei:

- erstens um die Beobachtung einer tief greifenden Veränderung der Sozialstruktur in ihrer funktionalen Differenzierung in Gestalt der Erosion der neokorporativen, sozialmarktwirtschaftlichen Institutionen zwischen Wirtschaft und Politik sowie der Ausbildung eines eigenständigen Handlungssystems Medien mit seinen Logiken und den damit verbundenen neuen Interdependenzen zwischen den Teilsystemen. Fokussiert wird insbesondere das Verhältnis zwischen dem politischen System, dem ökonomischen System und dem Mediensystem (Konzepte der Mediengesellschaft, Kommunikationsgesellschaft, Informationsgesellschaft und der Wissensgesellschaft). ${ }^{8}$

- Zweitens um die Beobachtung einer tief greifenden Veränderung der Sozialstruktur in ihrer stratifikatorischen Differenzierung durch neue Ungleichheiten hinsichtlich

8 Postman 1985; Willke 1992; Lash/Urry 1994; Münch 1995; Bell 1996; Knorr-Cetina 1999; Meier 2004, 57-78; Saxer 2004, 159-174. 
ökonomischen, sozialen und kulturellen Kapitals sowohl im Zentrum als auch in der Peripherie sowie zwischen Zentrum und Peripherie, verbunden mit einer neuen Fragilisierung der Erwartungssicherheit hinsichtlich Betätigungs- und Einkommenschancen in einer neuen desintegrierten Peripherie in den Zentrumsgesellschaften bis hin zu wachsenden Prekaritäten im Mittelstand durch die rechtliche und moralische Entformalisierung der Arbeitsbeziehungen (u. a. Prekarisierungsforschung, Bewegungsforschung und Konzepte der Weltgesellschaft, Weltsystemdebatte). ${ }^{9}$

- Drittens um die Beobachtung einer tief greifenden Veränderung der Sozialstruktur in ihrer segmentären Differenzierung durch die äußere und innere Tangierung des nationalstaatlichen Ordnungsprinzips der Weltgesellschaft in Gestalt einer Globalisierung der Wirtschaft und einer neuen transnationalen ,Kabinettspolitik' (G8, WTO, IMF, WEF etc.) mit wachsenden Legitimitätsdefiziten dieser supranationalen Institutionen einerseits sowie wachsenden Steuerungs- und Legitimitätsdefiziten der staatlichen politischen Institutionen andererseits. Zudem zeigt sich die segmentäre Differenzierung in der Semiperipherie und in der Peripherie in einer Renaissance des religiösen und ethnischen Bürgerkriegs, im Zerfall von Staaten (Rumpfstaaten) und der gleichzeitigen Re-Aktivierung tribaler Strukturen mit ihren Ehrbegriffen und Sanktionspotenzialen. In die Zentrumsnationen findet derweil ein migrationsbedingter Spannungstransfer statt, der insbesondere im Zusammenhang mit der Desindustrialisierung mit neuen Segregationseffekten entlang ethnisch-religiöser $\mathrm{Zu}-$ gehörigkeiten verbunden ist (Konzepte der Weltgesellschaft, Mediengesellschaft, transnationale Öffentlichkeit; Informationsgesellschaft, Migrationsgesellschaft und Minderheitengesellschaft). ${ }^{10}$

Wenn wir davon ausgehen, dass wir es hier mit den zentralen zeitgenössischen Differenzierungen zu tun haben, dann muss dies Anlass sein, die in der Kommunikationswissenschaft seit der zweiten Hälfte der 1990er Jahren reiche, jedoch funktionalistisch verkürzte Auseinandersetzung über Mediengesellschaft und Medialisierung zu öffnen. Hierzu drängt sich eine Argumentationsstrategie auf, die am Öffentlichkeitsbegriff ansetzt, also bereits schon eine Perspektive einnimmt, die den gesellschaftstheoretischen Anspruch mit sich führt. Mediengesellschaft und Medialisierung können dann auf einen „neuen“ Strukturwandel der Öffentlichkeit zurückgeführt werden, der in der Tat zur Ausdifferenzierung eines Handlungssystems Medien, aber auch zu neuen Kommunikations- und Rezeptionsschichtungen und -segmentierungen geführt hat. Dazu kann sich dieser Beitrag auf Münchs Auseinandersetzung mit diesem Strukturwandel (1995) sowie auf eine Reihe von Sammelbänden stützen, die sich aus kommunikationswissenschaftlicher Perspektive auf die Medien- oder Informationsgesellschaft und/oder auf Medialisierungseffekte konzentrieren und dabei einen wachsenden sozialtheoretischen Erklärungsanspruch erheben. ${ }^{11}$ Anschließend werden dann die in der Sozialtheorie dis-

9 Wallerstein 1979; Bourdieu 1987, 1998; Featherstone 1990; Balibar/Wallerstein 1991; Robertson 1992; Albrow 1996; Imhof/Eberle 2005.

10 Leggewie/Baringhorst 1990; Welsch 1995; Gerhards/Rössel 1999, 325-344; Imhof 2002, 37-56; Tobler 2004, 231-261; Neidhardt 2005.

11 Donsbach/Jarren/Kepplinger/Pfetsch 1993; Neidhardt 1994; Jarren 1994; Imhof/Schulz 1996; Jarren/Schatz/Weßler 1996; Sarcinelli 1998; Jarren/Sarcinelli/Saxer 1998; Imhof/Jarren/Blum 1999, 2002; Jarren/Imhof/Blum 2000; Bentele/Brosius/Jarren 2003; Imhof/Blum/Bonfadelli/ Jarren 2004, 2006. Die Perspektive in der genannten und der im Folgenden noch zitierten Literatur ist ausgesprochen stark auf die Interdependenz von Politik und Medien konzentriert. Vgl. hierzu auch: Theis-Berglmair/Bentele bzw. Selbstverständnisausschuss der DGPuK (2001): Die Mediengesellschaft und ihre Wissenschaft. Herausforderungen für die Kommunikations- und 
kutierten stratifikatorischen und segmentären Differenzierungsprozesse einbezogen, die im Rahmen der Kommunikationswissenschaft noch wenig Beachtung finden, gleichwohl aber Auswirkungen auf Kommunikation und Rezeption sowie Differenzierung und Integration haben.

Für diese Diskussion der Differenzierungsdynamiken ist eine kurze Reflexion auf die Bedingungen der Integration und der Steuerungsfähigkeit der modernen Gesellschaft zweckmäßig. An diesem Maßstab lassen sich dann die wichtigsten Probleme thematisieren, die durch die jüngsten Differenzierungsdynamiken ausgelöst werden: Demokratische Selbststeuerung impliziert, dass Gesellschaften auf sich selbst einwirken können. Um dies zu können, ist erstens die Geltung der Bürgerrechte Voraussetzung. Zweitens braucht es für die Bürgerinnen und Bürger einen politischen Begriff ihrer Gesellschaft und damit einen als gemeinsam erkannten politischen Geltungsbereich. Drittens braucht es ein politisches System, das in der Lage ist, über legitime Entscheidungen in diesem Geltungsbereich Ordnungslücken zu bearbeiten. Der Wert Demokratie impliziert schließlich viertens, dass sich die Bürgerinnen und Bürger im Sinne Kants als Autoren jener Gesetze und Institutionen betrachten können, denen sie sich selbst unterwerfen (Habermas 1992, 399-467). Alle diese Bedingungen setzen eine öffentliche Kommunikation voraus, die die Gesellschaft überwölbt, deren Arenen also wechselseitige Anschlusskommunikation schaffen, die deliberative Qualitäten besitzt und die - analog zur Sozialisationsagentur Bildungssystem - keine systematischen Ausschlüsse produziert. Erst eine solche Öffentlichkeit macht das, was wir in politischem Sinne Gesellschaft nennen, beobacht- und gestaltbar. Nur dies garantiert, dass sich soziale Differenzierungen, die in den politisch zentralen Fragen des guten Lebens immer zur Geltung kommen, dergestalt manifestieren können, dass die öffentliche Kommunikation ihre seismographische Funktion der Selektion politisch zu bearbeitender Probleme rechtzeitig erfüllen kann. Öffentliche Kommunikation ist somit das Medium der Integration wie der Selbststeuerung der Gesellschaft. In ihr kandidieren permanent Problematisierungen des guten Lebens um Aufmerksamkeit. In diesen Problematisierungen manifestieren sich die Wahrheits-, Gerechtigkeits- und Wahrhaftigkeitsansprüche von Selbst- und Fremdbeschreibungen, die sozialstrukturelle und kulturelle Differenzierungen abbilden, erzeugen, verstärken, mindern oder überdecken (Imhof 2006 [1996]).

Diese Voraussetzungen demokratischer Selbststeuerung, d. h. die Geltung der Bürgerrechte, die Verschränkung von politischem Geltungsbereich und politischer Öffentlichkeit, ein funktionierendes und legitimes politisches System sowie ausreichende Partizipationsdispositionen und eine öffentliche Kommunikation von seismographischer Qualität, ließen sich bisher nur im Rahmen des Nationalstaats einigermaßen realisieren. In diesem Ordnungsprinzip vollzog sich die Verbindung des Rechtsstaates mit dem Konzept der Nation, d. h. der symbolischen Konstruktion des Nationalstaates. Mit diesem Prozess einher ging die Herausbildung einer primär medienvermittelten Öffentlichkeit, die sich auf eben den mit dem Nationalstaat territorial definierten politischen Geltungsbereich mitsamt seinen Bürgerinnen und Bürgern bezieht (Kaelble et al. 2002).

Medienwissenschaft als akademische Disziplin. Selbstverständnispapier der Deutschen Gesellschaft für Publizistik- und Kommunikationswissenschaft (DGPuK) vom Januar 2001. Verfasst wurde das Papier vom Selbstverständnisausschuss unter Leitung der beiden Genannten, wird aber bereits wieder von neuen Selbstverständnisversuchen relativiert. 


\section{Neuer Strukturwandel der Öffentlichkeit}

In seinem „Strukturwandel der Öffentlichkeit“ postulierte Jürgen Habermas (1990 [1962]) die Verschränkung der Sphären Öffentlichkeit und Privatheit und beschrieb den politischen Funktionswandel der Öffentlichkeit zu einer massenmedial „hergestellten“ Öffentlichkeit. Er unterschied einen sozialen Strukturwandel und einen politischen Funktionswandel der Öffentlichkeit. Bezüglich ersterem postuliert er eine Verschränkung der im bürgerlichen Öffentlichkeitsverständnis konstitutiven Trennung der öffentlichen und der privaten Sphäre durch die wachsenden staatlichen Eingriffspotenziale in die Wirtschaft (und umgekehrt) und - damit verbunden - einen sukzessiven Bedeutungsverlust der orientierungsstiftenden Wirkung der Klassenschranken durch die Integration der Arbeiterbewegung in das sozialmarktwirtschaftliche Gesellschaftsmodell. Die Öffentlichkeit entgrenzt sich dadurch vom Bürgertum, und damit verliert der bildungsbürgerliche Diskurshabitus an Bedeutung. Entsprechend konstatiert Habermas eine Entwicklung vom kulturräsonierenden zum kulturkonsumierenden Publikum im Zuge seiner Erweiterung und Heterogenisierung (insbesondere durch Wahlrechtsreformen) (Habermas 1990, 225-274).

Korrelativ zu dieser Verschränkung der Sphären Öffentlichkeit und Privatheit bestimmte Habermas den politischen Funktionswandel der Öffentlichkeit als Entwicklung von den Versammlungsöffentlichkeiten der Aufklärungsbewegung und den diese Versammlungsöffentlichkeiten integrierenden Periodika hin zu einer massenmedial „hergestellten“ Öffentlichkeit. Diese löst sich vom Publikum ab und wird durch Staat, Parteien und insbesondere durch die organisierten Privatinteressen der Wirtschaft okkupiert und „vermachtet“. Dieser Vermachtungsprozess bedeutet ihm die Elimination des im Idealtypus bürgerlicher Öffentlichkeit angelegten emanzipativen Gehalts ( $\mathrm{Ha}$ bermas 1990, 275-342). In Anlehnung an die Ausdifferenzierung der bürgerlichen Öffentlichkeit aus der „repräsentativen Öffentlichkeit“ (Habermas 1990, 58-67) der anciens régimes interpretierte Habermas diese Vermachtung als Refeudalisierungsvorgang (Habermas 1990, 292, 337). Der Austritt aus der selbstverschuldeten Unmündigkeit via Deliberation führt auf diese Weise zurück in die Unmündigkeit einer bloß repräsentierenden und inszenierenden - eben feudalen - Öffentlichkeit im „Spätkapitalismus“. In diesem kulturindustriellen Entdifferenzierungsprozess von Staat, Parteien und organisierten Privatinteressen mitsamt den Seinsordnungen Öffentlichkeit und Privatheit verlieren die sozialstrukturellen Differenzierungen ihre sozialpolitische Energie, ihre Thematisierung erodiert, die Moderne reproduziert hinter diesem Verblendungszusammenhang bloß noch ihre Sozialstrukturen.

Auf der Linie dieser Diagnose ließen sich Ende der 1950er Jahre und in den frühen 60er Jahren die "zivilgesellschaftlichen Akteure“ mit ihrer Problematisierung der stratifikatorischen und segmentären Differenzierung der 1960er und 70er Jahre nicht erwarten. Unübersehbar wurzelt Habermas' damalige Sozialtheorie im Kulturindustriekapitel der Dialektik der Aufklärung und spiegelt Formen repräsentativer Öffentlichkeit im sozialpolitisch befriedeten und antikommunistisch integrierten sozialmarktwirtschaftlichen Gesellschafts- und Wirtschaftsmodell in den 1950er und frühen 60er Jahren.

Die Dynamik des „neuen“ Strukturwandels der Öffentlichkeit bricht sich erst danach Bahn. Dieser lässt sich als Auflösung des ,Vermachtungszusammenhangs' von Staat, Parteien und organisierten Privatinteressen im neuen neoliberalen Wirtschafts- und Gesellschaftsmodell beschreiben (Imhof/Eberle 2005). Im Kern handelt es sich bei diesem Vorgang, dessen deutliche Akzeleration in den 1980er Jahren zu beobachten ist, um die 
Deregulation der keynesianisch orientierten, neokorporativen Integration von Politik und Wirtschaft hin zu einem ökonomistisch argumentierenden Antietatismus im Steuer- und Standortwettbewerb und in diesem Kontext um die Deregulation der Medien von ihren politischen und sozialen Bindungen. Dieser Prozess hat die Erosion der gesinnungsethisch gebundenen Parteimilieus (Sarcinelli/Wissel 1998, 408-427; Schenk 1998, 387-407) in der programmatischen Annäherung der Volksparteien im Zeichen des globalen Dualismus des Kalten Krieges zur Voraussetzung (Imhof et al. 1996, 1999) und führt zur Entbettung der Medienorganisationen (Giddens 1992; Jarren 1994, 23-34), d. h. zur sozialen und ökonomischen Ablösung der Medien von ihren herkömmlichen, sozialräumlich und -moralisch gebundenen Trägern (Parteien, Verbände, Kirchen), zur Abkoppelung des Verlagswesens von den sozialmoralisch verankerten Netzwerken einer städtisch-bürgerlichen Elite und zur Umstellung familien- und sozialräumlich gebundener Kapitalversorgung privatrechtlicher Medienunternehmen auf beliebiges Investitionskapital mitsamt den damit verbundenen Konzentrationsprozessen (Jarren 1994, 23-34; 2001, 10-19; Siegert 2003, 20-30). Systemtheoretisch lässt sich dieser Vorgang wie folgt beschreiben:

- als Ausdifferenzierung eines eigenlogischen Mediensystems und die Koppelung der Medien an die Marktlogik; ${ }^{12}$

- als Ablösung des Staatsbürgerpublikums durch ein Publikum von Medienkonsumenten, um das auf der Basis von funktional, stratifikatorisch und segmentär differenzierenden Zielgruppenkonzeptionen geworben wird;

- als Entflechtung der medial erschlossenen Räume von den politischen Geltungsräumen;

- als Durchdringung der öffentlichen Kommunikation durch neue Selektions-, Interpretations- und Inszenierungslogiken.

Dieser, neben der Erosion der neokorporativen Interdependenzen von Politik und Wirtschaft, wichtigste Deregulationsvorgang im neoliberalen Wirtschafts- und Gesellschaftsmodell produzierte neue Medienorganisationen. Diese werden zu Dienstleistungsunternehmen mit beliebiger Kapitalversorgung und hohen Renditeerwartungen (Picard 2001, 65-69). Dadurch unterliegen sie einem raschen technischen Wandel, der sie durch die Nutzung der Konvergenzpotenziale der Massen- und Individualkommunikation in den ,Cyberspace' hineinführt, und sie werden ideologisch offener und flexibler (Kepplinger 1992). Sie generieren ihre eigenen Publika und orientieren ihre Selektions-, Interpretations- und Inszenierungslogiken über trial und error und angewandte Publikumsforschung an deren Aufmerksamkeitsbedürfnissen. Sie generieren ihre eigenen Produkte und Inhalte auf der Basis entsprechender Zielgruppenkonzeptionen (Hasebrink 1997, 262-280; Siegert 1998, 48-58); sie kreieren eine eigene Zeit, indem sie sich am wettbewerbsbedingten Aktualitätstempus orientieren (Sarcinelli 1994, 35-50; Saxer 1998, 21-6); und sie vernetzen Metropolen und erschließen kommunikativ neue Regionen. Im Zuge dieser sozialen und ökonomischen Autonomisierung unterliegen die

12 In der funktionalistisch argumentierenden kommunikationswissenschaftlichen Literatur lässt sich besonders im Rahmen systemtheoretischer Theoriekonzeptionen (Marcinkowski 1993; 2000, 49-73; Luhmann 1990, 170-182; 1996) theoriekonsistent eine Tendenz erkennen, neben dem Ausdifferenzierungsprozess des Mediensystems aus dem politischen System den korrelativen Entdifferenzierungsprozess, welcher die Medien der ökonomischen Marktlogik unterwirft, zu übersehen. In diesem Sinne ist auch der Begriff des Mediensystems als ,intermediäres System “ bei Gerhards/Neidhardt (1990, 90-101) einseitig durch die Vorstellung der Entkoppelung der Medien vom politischen System geprägt. 
Medienorganisationen einem raschen Wachstums- und Konzentrationsprozess (Knoche 1997, 123-158; Müller-Doohm 1998, 471-486). Die sozialräumliche Gliederung dieser Informationsökonomie folgt einem Transnationalisierungspfad (Gerhards/Rössel 1999, 325-344; Meier/Trappel 2005), der städtische Zentren mit Ballungsgebieten neu verknüpft, d. h. neue Sozialräume generiert und die territorial gebundenen politischen Institutionen einem Wettbewerb um Steuervorteile und Infrastrukturbedingungen aussetzt (Schwengel 1997, 663-678; Siegert et al. 2005, 469-494; Heinrich/Lobigs 2006).

Die aufgrund der seismographischen Funktion öffentlicher Kommunikation gesellschaftspolitisch wichtigsten Effekte dieses neuen Strukturwandels im deregulierten Dreieck Politik, Medien und Ökonomie ergeben sich über die Veränderungen der medienvermittelten Kommunikation. Diese wurden bisher noch wenig in Form von aussagekräftigen Zeitreihenanalysen und mit Bezug auf die notwendigen Anpassungsleistungen der Organisationen des politischen oder ökonomischen Handlungssystems an die Inputbedürfnisse der Medien untersucht (Medialisierungseffekte). ${ }^{13}$ Anhand der vorhandenen Forschungsergebnisse lässt sich eine Reihe von Indikatoren gewinnen, die insbesondere seit den 1980er Jahren tief greifende Veränderungen in der öffentlichen Kommunikation anzeigen. In ihnen widerspiegeln sich Dynamiken, welche die Allokation von Aufmerksamkeit, Definitionsmacht und Reputation in funktionaler, stratifikatorischer und segmentärer Hinsicht neu organisieren:

Erstens kann seit den 1970er Jahren eine Verstetigung des Phänomens sozialer Bewegungen und Protestparteien sowie die Institutionalisierung resonanzorientierter NGOs beobachtet werden. Im diachronen Vergleich der Krisen von Gesellschaftsmodellen, in denen nicht-etablierte Akteure regelhaft eine zentrale Rolle spielen, ist die Lebensdauer dieser ,neuen' sozialen Bewegungen erstaunlich lang, und sie eroberten ganze politische Themenbereiche wie Umwelt- und Technikfolgeprobleme, Sicherheitspolitik, Geschlechterdifferenz, Migrations- und Asylpolitik für sich (Rucht 1994, 337-358). In der Katastrophen- und Risikokommunikation gelang es ihnen, eine neue MenschUmwelt-Beziehung zu implementieren, die sich sowohl im Rechtsetzungsprozess wie im Alltagshandeln niederschlug. Dasselbe gilt für die Veränderungen der Geschlechterrollen und für die Ergänzung des Ost-West-Dualismus durch den Nord-Süd-Dualismus. Schließlich implementierten diese nicht-etablierten Akteure auch den ausgeprägten Antietatismus, der das sozialmarktwirtschaftliche Gesellschafts- und Wirtschaftsmodell schon vor der neoliberalen Semantik der Globalisierung und des Steuer- und Standortwettbewerbs schwächte (Boltanski/Chiapello 2003 [1999], 211-260). Die gegenüber den 1950er und frühen 60er Jahren sprunghaft erhöhte Resonanz nicht-etablierter politischer Akteure in den 1970er und 80er Jahren kann zum einen krisen- und konflikttheoretisch als Regularität in der Abfolge von Gesellschaftsmodellen erklärt werden (Imhof 2003, 153-182). Die Konstanz dieses Phänomens muss jedoch zum anderen auch auf den neuen Strukturwandel der Öffentlichkeit zurückgeführt werden, der den medienwirksamen Aktionsformen nicht-etablierter Akteure wesentlich bessere Resonanzchancen vermittelt als die noch verstärkt durch (partei-)politische Selektions- und Interpretationslogiken gesteuerte öffentliche Kommunikation zuvor (Rucht 2003, 411). Inzwischen lassen sich ein Rückgang der Resonanz nicht-etablierter Akteure und ihre Institutionalisierung in etablierte Parteien oder NGOs konstatieren. Die These vom

13 Altheide/Snow 1979; Kaase 1998, 24-51; Pfetsch 1998, 406-413; Blumler/Gurevitch 2001, 380 403; Holtz-Bacha 1999; Donges 2000; Bennett/Entman 2001; Kepplinger 2002, 972-986; Krotz 2002, 168-183; Kamber/Schranz 2002, 347-363; Donsbach/Jandura 2003; Kamber/Imhof 2004, 10-18; Schulz 2004, 87-101. 
Einritt in die „Bewegungsgesellschaft“ (Neidhardt/Rucht 1993) muss modifiziert werden. Die damit verbundenen Erwartungen an „zivilgesellschaftliche Assoziationen“, die der Deliberation dauerhaft eine neue Qualität verleihen, erfüllen sich nicht (Habermas 1992, 386).

Bei den Gründen hierfür handelt es sich zunächst um einen deutlichen Wandel der politischen Kommunikation von Seiten etablierter Akteure. Zu beobachten ist ein Transfer von Techniken des Aufmerksamkeitsmanagements, d. h. es lassen sich eine Zunahme medienwirksamen „Eventmanagements“ (Kepplinger 1992; Schmitt-Beck/ Pfetsch 1994, 106-138; Imhof/Eisenegger 1999, 195-218), eine ausgesprochene Personenzentrierung und eine deutlich gestiegene Bedeutung von Konfliktinszenierungen feststellen. Wir haben es bei den Kommunikationsformen der etablierten Akteure mit erfolgreichen, nachrichtenwertorientierten Anpassungen an die Selektions-, Interpretations- und Inszenierungslogiken der Medien zu tun. Damit haben die etablierten Akteure einen Vorteil der nicht-etablierten Akteure im Wettbewerb um Aufmerksamkeit egalisiert. Im Rahmen dieser Neuallokation der Resonanzchancen lässt sich außerdem ein Bedeutungsgewinn charismatischer Beziehungen in der Politik beobachten sowie die Etablierung eines neuen Parteientyps, der sich als „Bewegungspartei“ definieren lässt. Insbesondere rechtsbürgerliche Bewegungsparteien mit schwachen Strukturen, niederen Partizipationsschwellen und charismatischer Führung konnten und können in vielen europäischen Ländern das traditionelle Parteiengefüge sprengen, indem sie äußerst medienwirksam und thematisch fokussiert vorab über eine variantenreiche Problematisierung des Fremden (in Form von Immigranten wie von ,Brüssel' ${ }^{`}$ ) einerseits, einem ausgeprägten Antietatismus andererseits, Protest- und Wechselwähler binden und die Kontingenz des Politischen erhöhen (Imhof 1996, 165-186). Beobachtbar ist also eine aufmerksamkeitsorientierte thematische Fokussierung des Politischen, erhöhte Resonanzchancen nicht-etablierter politischer Akteure und von Bewegungsparteien, entsprechende Anpassungen etablierter Akteure sowie ein Bedeutungsgewinn inkludierender wie exkludierender Identitätspolitik.

Bei der medialen Skandalisierungskommunikation handelt es sich um einen aussagekräftigen zweiten Indikator des neuen Strukturwandels: Zeitreihenanalytische Untersuchungen von Skandalisierungen in der öffentlichen Kommunikation zeigen eine massive Zunahme des Phänomens. Bei dieser Intensivierung der Skandalkommunikation ist auch eine Veränderung der tripolaren Struktur des Skandals beobachtbar: Während der klassische Skandal aus einem Skandalisierer, einem Skandalmedium und einem Skandalisierten besteht, übernimmt nun das Skandalmedium in der Regel auch die Rolle des Skandalisierers (Neckel 1986, 581-605; Kepplinger 1996, 41-58; Imhof 2000, 55-68; Hondrich 2002). Seit den 1980er Jahren hat sich auf der Basis dieses intermedialen Aufmerksamkeitswettbewerbs eine effiziente, empörungsbewirtschaftende Expertenkultur der Skandalisierung ausdifferenziert, die medienexterne Skandalisierer substituiert und damit auch eine zentrale Funktion nicht-etablierter politischer Akteure professionalisiert. Besonders auffällig ist die Veränderung in der Katastrophen- oder Risikokommunikation. Tradierte Deutungsmuster wie Schicksal, Zufall oder Unglück werden durch personalisierte Verantwortungszuordnungen abgelöst. Obwohl nach wie vor Skandalisierungen gegenüber Vertretern des politischen Personals überwiegen, lässt sich feststellen, dass die Skandalisierung von Unternehmen mitsamt ihrem Führungspersonal drastisch zunimmt (Eisenegger 2005). Dabei kommt die resonanzstärkste Skandalisierung der Wirtschaft nicht mehr von Verbänden und Parteien, sondern von Medienorganisationen. Zudem erweitern sich die skandalisierungsfähigen Themen. Moralische Verfehlungen, die der privaten Sphäre entstammen, wurden öffentlichkeitsfähig. Beob- 
achtbar ist entsprechend eine ausgeprägte moralische Aufladung der öffentlichen Kommunikation. Die alarmistische Empörungsbewirtschaftung ist zu einem zentralen Mittel des Aufmerksamkeitswettbewerbs geworden. Die Ausdifferenzierung des Mediensystems hat somit zur Genese eines neuen politischen Akteurs geführt, der nachrichtenwertorientiert größtmögliche Normverstöße hochpersonalisiert bearbeitet. Diese finden sich in den stratifikatorisch relevanten Dimensionen Machtmissbrauch und Vorspiegelung falscher Tatsachen insbesondere des politischen und ökonomischen Führungspersonals sowie in segmentär relevanter Hinsicht im Rassismus- und Fremdenfeindlichkeitsvorwurf. Gleichzeitig wird das kriminelle Verhalten von Angehörigen von Immigrationspopulationen stärker mit Herkunftshinweisen versehen.

Drittens ist im historischen Vergleich eine beispiellose Privatisierung des Öffentlichen und Personalisierung der politischen Kommunikation zu konstatieren (Imhof 1999, 717-732). Beobachtbar ist eine Zunahme von Human Interest Storys, Betroffenheits-, Thesen- und Moraljournalismus und die Etablierung neuer boulevardisierender Nachrichtenformate in Radio und Fernsehen. Die Personalisierung des Politischen findet im Fernsehen ihr wichtigstes Medium: Politikdarstellung gleicht sich strukturell und inhaltlich der Unterhaltung an, und politische Argumente werden durch Charakterdarstellungen im privaten Lebensraum und medienattraktive Konfliktinszenierungen ergänzt (Sarcinelli 1987, 1994; Schmitt-Beck/Pfetsch 1994, 106-138; Jarren 1996, 79-96; 1998, 74-96). Dadurch zeigt sich im diachronen Vergleich ein Wandel in der medialen Kreation von Prominenz, die Ausbildung transmedialer Verwertungsketten von Prominenten und neue Reputationsdynamiken. Die Darstellung von Vertretern des politischen Personals gleicht sich der Darstellung der Gesellschaftsprominenz an: Neben dem Machtstatus entscheiden die telegene Inszenierung privater Lebensstile und Selbstdarstellungskompetenzen über mediale Resonanz (Wilke 1996, 99-106). Durch Ereignisproduktion (etwa in Gestalt von Homestorys) wirken die medialen Inszenierungsmuster in die Personalauswahl der Teilsysteme ein und schaffen in der Politik (wie in der Wirtschaft) ein Starsystem (Peters 1994, 191-213). Entsprechend hat die Kommunikation von Gruppenpositionen auf Seiten der Medien wie auf Seiten der Vertreter des politischen Personals stark abgenommen. Politische Positionen werden kommunikativ immer mehr Individuen, nicht Parteien oder Verbänden zugeordnet und mit Charakterdarstellungen zur Inszenierung von Authentizität und Integrität verknüpft. Diese neuen Formen der Politikvermittlung stellen angesichts der Erosion der Parteimilieus eine neue Komplexitätsreduktion dar, indem sie den Entscheidungsfindungsprozess auf Sympathie oder Antipathie und damit auf die affektive Komponente personaler Reputation umstellen. ${ }^{14}$ Vergleichbares lässt sich in der kommunikativen Konstitution der Ökonomie beobachten. Die gewachsene Organisationsreputation von Unternehmen wird durch die personale Reputation des Führungspersonals überformt. Damit erhöht sich die Volatilität der zentralen Ressource Reputation und das Risiko des Reputationsverlusts. Sowohl im politischen als auch im ökonomischen Handlungssystem bestimmt die medienvermittelte Reputationsallokation die hergebrachten Karrierewege, bzw. politische und ökonomische Organisationen müssen bei der Auswahl ihrer Hierarchiespitzen systemfremde Faktoren berücksichtigen.

Als vierten Indikator des Strukturwandels lässt sich eine deutliche Verschiebung in

14 Ironischerweise verliert dabei die hergebrachte kognitiv-funktionale und asketische Reputationskomponente ,Leistung' ausgerechnet in der Leistungsgesellschaft an Bedeutung. In Wahlkämpfen wird Leistung in der medienvermittelten öffentlichen Kommunikation inzwischen hauptsächlich am Erfolg im Resonanzwettbewerb gemessen. 
der intermedialen Themen- und Meinungsresonanz feststellen. Die Parteipresse bzw. die parteinahe Gesinnungspresse war gekennzeichnet durch eine ausgeprägte wechselseitige Themen- und Meinungsresonanz, weil der Kampf um die öffentliche Meinung durch Weltanschauungsorgane bestritten wurde, deren Redaktionen die intellektuellen Speerspitzen der Parteien darstellten. Diese nahmen sich wechselseitig als pars pro toto des politischen Gegners wahr und vermittelten die parlamentarische Auseinandersetzung zum Staatsbürgerpublikum. Mit der Erosion dieses publikumsoffenen Streits zwischen Leitmedien verkürzt sich die Themen- und Meinungsresonanz auf eine bloße Themenresonanz, und der deliberative Kernbereich des politischen Systems, das Parlament, findet in der medienvermittelten politischen Kommunikation zu Gunsten der Exekutive immer weniger Resonanz. Trotz der Zunahme der thematischen Referenzialität zwischen den Leitmedien innerhalb einer Arena ist somit eine Abnahme der Anschlusskommunikation zu verzeichnen. Mit diesem Schwund des Meinungsstreits in und zwischen politisch profilierten Medien erodiert die Deliberation, und das Parlament wird zugunsten einer am Nachrichtenwert ,Herrschaftsposition“ orientierten Fokussierung auf die Exekutive kommunikativ isoliert (Negrine 1998; Marcinkowski 2000, 49-73; Imhof/Kamber 2001, 425-453; Ettinger 2005, 75-90). Gemessen an der tradierten Form der Selektion von Problemen, die um Eingang in den politischen Entscheidungsfindungsprozess kandidieren, lässt sich eine Entwertung des Parlaments zugunsten der Exekutive und der medienvermittelten Kommunikation feststellen. Auch etablierte politische Akteure versuchen verstärkt, über eine medienvermittelte Problemakzentuierung das politische System von außen zu beeinflussen.

Damit zeichnet sich fünftens eine Umkehrung der Vermittlungslogik der politischen Kommunikation ab. Für die Prioritätenordnung politischer Probleme werden die Selektions- und Interpretationslogiken des Mediensystems wichtiger. Die Aufmerksamkeitsregeln resonanzorientierter Politik drücken auf die Entscheidungspolitik durch (Sarcinelli 1994). Dies bedeutet, dass die Medienorganisationen selbst - und das politische Personal über die Medien - Einfluss auf die Agenda des politischen Systems erzielen (Schranz 2000). In diesem Zusammenhang sind Kampagnenpartnerschaften zwischen Parteien und Medienorganisationen beobachtbar. Auf der Basis von redaktionellen Leitlinien sowie politischen Orientierungsvorgaben für ganze Medienorganisationen zeichnet sich inzwischen eine volatile, von Besitzverhältnissen und Marktanalysen abhängige Repolitisierung der Forumsmedien ab. Dadurch haben wir es mit einem medienplebiszitären Druck und einer „mediendramaturgischen Umwertung“ - zumeist über die Selektion politischer Konflikte und ihre Akzentuierung - und darüber hinaus mit einer intensiven Ereignis- und Kampagnenproduktion von Seiten der Medien zu tun (Münch 1997, 696-709). Jede Beschreibung der medienvermittelten Kommunikation, die nur die Selektionsleistungen im Blick hat (gate keeper), zielt an der medialen Konstruktion der Wirklichkeit vorbei. Auch der publizistikwissenschaftliche Begriff des Agenda Setting wird dieser eigenständigen Ereignisproduktion nicht mehr gerecht (vgl. etwa Schmolke 1996, 187-200; Blum 1996, 201-212).

Bereits diese stark auf die Interdependenzen von Politik, Medien und Wirtschaft beruhende Diskussion von Indikatoren des neuen Strukturwandels der Öffentlichkeit, zeigt noch ohne Rekurs auf die Rezeptionsebene, dass die funktionale Ausdifferenzierung des Mediensystems auch stratifikatorische und segmentäre Differenzierungseffekte zeitigt. Wenn nun noch die im ersten Anschnitt erwähnten Differenzierungsdynamiken berücksichtigt werden, dann lässt sich ein Begriff der Mediengesellschaft erarbeiten, der Anschluss an die sozialtheoretische Zeitdiagnostik findet. Ein solcher kommunikationswissenschaftlicher Zugang öffnet die Gegenstandsfokussierung auf die Massenme- 
dien; er muss sich dann allerdings auch der sozialwissenschaftlichen Frage einer funktionierenden sozialen Ordnung stellen.

\section{Fazit: Mediengesellschaft und Medialisierung}

Die zeitgenössische Gesellschaft ist funktionalen, stratifikatorischen und segmentären Differenzierungsdynamiken und daraus hervorgehenden Medialisierungseffekten ausgesetzt, die sowohl ihre Sozialstruktur als auch die Bedingungen demokratischer Selbststeuerung und Integration tiefgreifend tangieren. Der Begriff der Mediengesellschaft ist deshalb auf alle Differenzierungsdimensionen abzustützen. Daraus leiten sich die $\mathrm{Me}-$ dialisierungseffekte $\mathrm{ab}$, die der kommunikationswissenschaftlichen Forschung bedürfen, um Orientierungswissen für die Gesellschaft bereitzustellen:

1. Die Mediengesellschaft in der Dimension funktionaler Differenzierung: Die Ausdifferenzierung eines eigenständigen Mediensystems verändert die kommunikative Infrastruktur der modernen Gesellschaft grundlegend, führt zu neuen ,entbetteten ' $\mathrm{Me}-$ dienorganisationen (Giddens 1992), neuen und gleichartigen Selektions-, Interpretations- und Inszenierungslogiken, einem Siegeszug der Visualität und des Narrativen, einer ausgeprägten Exekutivorientierung und einer massiven Zunahme der Skandalisierungskommunikation. Dieser Prozess führt zu neuen Interdependenzen zwischen den zentralen Teilsystemen Politik, Medien und Wirtschaft und deren Organisationen und hat organisationsinterne Ausdifferenzierungen und Rollenanpassungen zur Folge, weil die Parteien, parlamentarischen Fraktionen und Verbände mit ihren direkten Vermittlungsorganen auch ihre eigenen Publikumssegmente verlieren, während Regierung und Verwaltung mit einer Medienarena konfrontiert werden, die sich den Agenden der politischen Willensbildung und Entscheidung höchst selektiv annimmt, diese Agenden im Rahmen von Kampagnenjournalismus medienplebiszitär beeinflusst und die die auf Koordinations- und Verfahrensprozesse geeichte Politik unter ,Reaktionsstress' gegenüber dem medialen Aktualitätstempus setzt. Gleichzeitig verliert der hergebrachte Verlautbarungsjournalismus gegenüber der Ökonomie an Bedeutung, bzw. die Wirtschaftsberichterstattung gleicht sich den Selektions- und Interpretationslogiken der politischen Berichterstattung an. Die Medien schieben sich zwischen Politik und Wirtschaft. Insgesamt bedeutet dies eine Neukonstitution des primären Mediums der Steuerung und Integration moderner Gesellschaften: der öffentlichen Kommunikation.

2. Die Mediengesellschaft in der Dimension stratifikatorischer Differenzierung: Hier$\mathrm{zu}$ zählen die Folgen der nationalen und internationalen Konzentrationsprozesse im Medienwesen (Trappel et al. 2002), die weltweite Hierarchisierung der Leitmedien insbesondere bei Schlüsselkommunikationsereignissen, die Adaptionen des Politischen an plebiszitäre Medienmacht, die Ablösung klassischer Sozialisationsagenturen durch lebensstilgruppen- und szenespezifische Medien (Giddens 1991; Schulze 1993; McQuail 2000, 72; Winter et al. 2003) und die Akzentuierung der Ungleichheitsrelationen durch die Differenzierung des Medienangebots und der Mediennutzung gemäß unterschiedlichem ökonomischem, sozialem und kulturellem Kapital (Knowledge Gap bzw. Digital Divide, Kultivierungsanalyse, Media Malaise) (Bonfadelli 1994, 2002, 65-84; Viswanath/Finnegan 1996, 187-227; Wirth 1997; Norris 2001; Marr 2004, 76-94). Dieser Prozess wird akzentuiert durch eine migrationsbedingte Unterschichtung aus Immigrantenpopulationen ohne oder mit reduzierten politischen Rechten und Kommunikationsfähigkeiten bei deutlich intensivierter Versorgung durch ,Herkunftsmedien'. Zusätzlich sind an den Rändern der Zentrumsgesellschaften, insbesondere in den Jugendkulturen der Banlieues, neue, eigenständige und abgeschottete Kommunikationsarenen und se- 
kundäre Desintegrationsprozesse beobachtbar, die zu einer Reduktion der Chancengleichheit führen. Dies bedeutet unterschiedliche Zugangschancen zur politischen Öffentlichkeit und damit verbundene Wissens- und Partizipationsklüfte.

3. Die Mediengesellschaft in der Dimension segmentärer Differenzierung: Beobachtbar ist die äußere und innere Tangierung des nationalstaatlichen Ordnungsprinzips in Gestalt einer Entschränkung von politischem Geltungsbereich und medial erschlossenen Räumen. Dies gilt zunächst auf nationalstaatlicher Ebene, schafft ,entöffentlichte politische Institutionen in ökonomisch ertragsschwachen lokalen Räumen und kreiert - gemäß Absatzkriterien - medial neu erschlossene Räume ohne Bezug zu (föderalen und nationalen) politischen Geltungsbereichen. Auf europäischer Ebene verhält es sich umgekehrt: Die politischen Institutionen der Europäischen Union sind in der medienvermittelten Kommunikation nur das Kaleidoskop nationalstaatlicher Bezüge (Gerhards 1993, 96-110). Auf internationaler Ebene etablieren sich besonders im Kontext der ökonomischen Globalisierung neue politische Institutionen ohne politische Öffentlichkeit (neue ,geheime Kabinettspolitik'). Die demokratienotwendige Verschränkung von politischem Geltungsbereich und Öffentlichkeit findet nicht statt; die Öffentlichkeit wächst der Globalisierung von Ökonomie und Politik nicht nach. Diese Entkoppelung von Politik und Öffentlichkeit innerhalb wie außerhalb des nationalstaatlichen Ordnungsrahmens widerspricht der notwendigen Bedingung für die Konstitution und Reproduktion eines (immer mehrschichtigen) Gemeinsamkeitsglaubens, der für die Akzeptanz von Mehrheits- und Minderheitenentscheiden und Umverteilungen Voraussetzung ist. Außerdem haben wir es mit einer migrationsbedingten Segmentierung der Öffentlichkeit entlang ethnisch-religiöser Zugehörigkeiten und Herkunftsbezügen zu tun (Heitmeyer et al. 1997; Hoffmann-Nowotny 2001; Meier-Braun/Kilgus 2002).

Diese Heuristik der Mediengesellschaft und der Medialisierungseffekte unter Beachtung aller Differenzierungsdimensionen lässt sich weiter differenzieren, indem die Makro-, die Meso- und die Mikroebene der sozialwissenschaftlichen Analyse einbezogen werden. Dies führt zu einer Kreuztabellierung, die es erlaubt, die bezüglich Differenzierung, Steuerungsfähigkeit und Integration der modernen Gesellschaft relevanten Forschungsfelder der Kommunikationswissenschaft darzustellen und die wichtigsten Lücken in der Medialisierungsforschung zu benennen (siehe Tabelle).

Wenn die Felder dieser Kreuztabellierung auf der Basis aller Differenzierungsdimensionen und der sozialwissenschaftlichen Zugangsebenen mit den bereits bearbeiteten Forschungsbezügen der Kommunikationswissenschaft verglichen werden, dann lässt sich erstens vergegenwärtigen, dass die Medialisierungseffekte der funktionalen Differenzierung auf der Makro-, der Meso- und der Mikroebene in der zeitgenössischen kommunikationswissenschaftlichen Forschung am meisten Beachtung finden. Auf der Basis dieser Differenzierungsdimension trägt die Kommunikationswissenschaft maßgeblich zum Verständnis der modernen Gesellschaft bei, wenn auch weitgehend auf das Verhältnis von Politik und Medien fokussiert. Die PR-Forschung hat in dieser funktionalen Differenzierungsdimension bezüglich des Verhältnisses von Ökonomie und Medien noch nicht den Stand der kommunikationswissenschaftlichen Analyse des Verhältnisses von Politik und Medien erreicht: Hinsichtlich der Interdependenz zwischen medienvermittelter Öffentlichkeit und Wirtschaft verbleibt die PR-Forschung noch im Kanon ihrer traditionellen Fragestellungen zum Verhältnis von Organisationen und ihren Teilöffentlichkeiten und thematisiert die kommunikative Neukonstitution der Ökonomie kaum. Dies bedeutet, dass die PR-Forschung insbesondere die neuen Reputationslogiken bezüglich Unternehmen und ihres Führungspersonals, die Überformung der Organisationsreputation durch personale Reputation bzw. die Charismaeffekte bei 
Tabelle: Mediengesellschaft und Medialisierung gemäß Differenzierungsdimensionen und Zugangsebenenen

\begin{tabular}{|c|c|c|c|}
\hline & $\begin{array}{l}\text { Funktionale } \\
\text { Differenzierung }\end{array}$ & $\begin{array}{l}\text { Stratifikatorische } \\
\text { Differenzierung }\end{array}$ & $\begin{array}{l}\text { Segmentäre } \\
\text { Differenzierung }\end{array}$ \\
\hline $\begin{array}{l}\text { Makro- } \\
\text { ebene }\end{array}$ & $\begin{array}{l}\text { Ausdifferenzierung des } \\
\text { Mediensystems Neukonsti- } \\
\text { tution der politischen Kom- } \\
\text { munikation, Umkehrung } \\
\text { ihrer Vermittlungslogik, } \\
\text { mediendramaturgische Um- } \\
\text { wertung der politischen } \\
\text { Agenda, Adaptionen des } \\
\text { politischen Systems an die } \\
\text { medialen Selektions- und } \\
\text { Interpretationslogiken so- } \\
\text { wie an die gesunkene Mei- } \\
\text { nungsresonanz und die ge- } \\
\text { steigerte Exekutivfixierung. }\end{array}$ & $\begin{array}{l}\text { Weltkommunikations- } \\
\text { schichtung, Weltkommuni- } \\
\text { kationsgesellschaft: } \\
\text { „Global Divide“, Monopo- } \\
\text { lisierungseffekte, Globali- } \\
\text { sierung westlicher Stan- } \\
\text { dards und Erwartungs- } \\
\text { strukturen, Förderung von } \\
\text { Migrationsprozessen bei } \\
\text { reduzierten Integrations- } \\
\text { chancen durch die identi- } \\
\text { tätsreproduktiven Effekte } \\
\text { der Herkunftsmedien. }\end{array}$ & $\begin{array}{l}\text { Entgrenzung von politi- } \\
\text { schen Geltungsbereichen } \\
\text { und kommunikativ er- } \\
\text { schlossenen Räumen auf } \\
\text { transnationaler Ebene: Tan- } \\
\text { gierung des nationalstaatli- } \\
\text { chen Ordnungsprinzips } \\
\text { ohne medial erschlossene } \\
\text { politische Geltungsräume. } \\
\text { Souveränitätsverluste ohne } \\
\text { Souveränitätsgewinne: } \\
\text { transnationale Entöffent- } \\
\text { lichung. }\end{array}$ \\
\hline $\begin{array}{l}\text { Meso- } \\
\text { ebene }\end{array}$ & $\begin{array}{l}\text { Neue Interdependenzdyna- } \\
\text { miken zwischen politischen } \\
\text { Organisationen und Medi- } \\
\text { en: Strukturwandel der Or- } \\
\text { ganisationen (u. a. Bewe- } \\
\text { gungsparteien), Instrumen- } \\
\text { talisierung von Politik und } \\
\text { Medien; neue Auswahlprin- } \\
\text { zipien des Spitzenpersonals; } \\
\text { Stärkung der Organisa- } \\
\text { tionsspitze und Professio- } \\
\text { nalisierung der Kommuni- } \\
\text { kation; Widersprüche zwi- } \\
\text { schen der personalisierten } \\
\text { Außen- und der,wir-orien- } \\
\text { tierten' Binnenkommunika- } \\
\text { tion. }\end{array}$ & $\begin{array}{l}\text { Neuallokation der Defini- } \\
\text { tionsmacht auf der Basis } \\
\text { von Nachrichtenwerten, } \\
\text { Medienmacht und Kapital } \\
\text { sowie durch die unter- } \\
\text { schiedliche Professionalisie- } \\
\text { rung der Organisations- } \\
\text { kommunikation, medien- } \\
\text { plebiszitäre Effekte und } \\
\text { Ablösung klassischer Sozia- } \\
\text { lisationsagenturen durch } \\
\text { szenenspezifische Medien- } \\
\text { produkte. }\end{array}$ & $\begin{array}{l}\text { Entgrenzung von politi- } \\
\text { schen Geltungsbereichen } \\
\text { und kommunikativ er- } \\
\text { schlossenen Räumen auf } \\
\text { nationaler Ebene: Entöf- } \\
\text { fentlichung des Lokalen, } \\
\text { mediale Neuerschließungen } \\
\text { ohne Berücksichtigung po- } \\
\text { litischer Geltungsräume. In- } \\
\text { tensivierung sprachnational } \\
\text { und ethnisch orientierter } \\
\text { Medienangebote gegenüber } \\
\text { Migrantenpopulationen. }\end{array}$ \\
\hline $\begin{array}{l}\text { Mikro- } \\
\text { ebene }\end{array}$ & $\begin{array}{l}\text { Rollenspezialisierungen; } \\
\text { Professionalisierungen; Ins- } \\
\text { zenierungen (Privatisierung, } \\
\text { Personalisierung); Charis- } \\
\text { matische Beziehungen und } \\
\text { neue Reputations- und Pro- } \\
\text { minenzierungslogiken. }\end{array}$ & $\begin{array}{l}\text { Abschichtung der Partizi- } \\
\text { pationsmöglichkeiten: } \\
\text { „Digital Divide“, „Media } \\
\text { Malaise“; Partizipationsdif- } \\
\text { ferenzierung gemäß ökono- } \\
\text { mischen, sozialen und kul- } \\
\text { turellen Kapitalsorten, se- } \\
\text { kundärer Analphabetismus. }\end{array}$ & $\begin{array}{l}\text { Identitätskonzepte und Me- } \\
\text { dienkonsum; Individualisie- } \\
\text { rung als Segmentierung in } \\
\text { Gestalt von Lebensstilgrup- } \\
\text { pen und Subkulturen; se- } \\
\text { kundäre Ethnisierungspro- } \\
\text { zesse durch ethnisch orien- } \\
\text { tierte Herkunftsmedien. }\end{array}$ \\
\hline
\end{tabular}

der Managerelite, die moralische Aufladung der Wirtschaftsberichterstattung und alle damit verbundenen Medialisierungseffekte vernachlässigt. Bezüglich Medialisierungseffekten der funktionalen Differenzierung in anderen Teilsystemen ist die kommunikationswissenschaftliche Forschung generell noch sehr schmal. 
Cum grano salis zeigt diese Tabelle zweitens in der Richtung von links nach rechtsoben zunehmend diejenigen Forschungsfelder, die von der Kommunikationswissenschaft (noch) vernachlässigt werden, obwohl sie für die Steuerung und Integration der modernen Gesellschaft von ausschlaggebender Bedeutung sind. Die Dominanz der funktionalen Differenzierung führte zu diesem Balanceverlust in der Produktion von Orientierungswissen. Hinsichtlich der stratifikatorischen und segmentären Differenzierung gilt dies am wenigsten für die Mikroebene. Die Rezeptionsforschung interessiert sich traditionellerweise für stratifikatorische Effekte und erarbeitete u. a. mit der Wissenskluftforschung empirisch gestützte Heuristiken des Rezeptionsverhaltens und der Medienwirkung. Die Cultural Studies konzentrieren sich ausgesprochen stark auf die stratifikatorischen Effekte bei der Encodierung und Decodierung von Medieninhalten, und ihre Vertreter arbeiten auch intensiv an der diskursanalytischen Erforschung der identitätskonstitutiven sozialen Praxen segmentärer Vergemeinschaftungsformen.

Damit lässt sich drittens feststellen, dass die Forschungsfelder der stratifikatorischen und segmentären Differenzierung auf der Meso- und Makroebene jene Desiderata darstellen, denen sich die Kommunikationswissenschaft verstärkt zuwenden muss. Dies bezieht sich in der stratifikatorischen Dimension auf die Kommunikationsschichtung auf der Ebene der Weltgesellschaft und auf die Neuallokation der Definitionsmacht insbesondere der politischen Akteure, auf die Folgen der erhöhten Kommunikationschancen der Exekutive für die Gewaltenteilung und für die Veränderung des Policy Cycles bzw. demokratischer Entscheidungsfindungsprozesse durch medienplebiszitäre Macht sowie auf die neue Bedeutung von Medien als Sozialisationsagenturen für Lebensstilgruppen. Schließlich wird in der Kommunikationswissenschaft in der Dimension der segmentären Differenzierung die Entschränkung von politischen Geltungsräumen und medial erschlossenen Räumen - insbesondere auf transnationaler Ebene - vernachlässigt, obwohl dies die demokratische Selbststeuerung im Kern gefährdet. Die Forschung über transnationale Öffentlichkeiten ist schmal und praktisch ausschließlich soziologischer Provenienz. Auch auf der Mesoebene kommt der Entöffentlichung ökonomisch ertragsschwacher politischer Geltungsräume zu wenig Aufmerksamkeit zu. Dasselbe gilt für die ,Leopardisierung' der öffentlichen Kommunikation durch Herkunftsmedien für Immigrantenpopulationen. Demokratietheoretisch ist insbesondere bedenklich, dass die Herkunftsmedien die Agenda und die Kommunikationsfähigkeiten auch der zweiten und dritten Generation der Immigrantenpopulationen beeinflussen, während die politischen Rechte dieser Kohorten beschnitten bleiben.

Dieser Vorschlag eines Mediengesellschaftsbegriffs, der den Versuch macht, das differenzierungstheoretische Erbe umfassend in Anschlag $\mathrm{zu}$ bringen und Medialisierungseffekte in allen drei Differenzierungsdimensionen auf allen drei Ebenen sozialwissenschaftlicher Forschung zu berücksichtigen, hat den Vorteil, die Potenziale der Kommunikationswissenschaft für die Zeitdiagnostik umfänglich zu nützen und dem Fach entsprechende Geltung zu verschaffen. Außerdem lässt sich durch die Diskussion von Mediengesellschaft und Medialisierung auch die ausgeprägte Binnendifferenzierung des Faches in Kommunikator-, Vermittlungs- und Rezeptionsforschung durch einen gemeinsamen Bezug zu den zentralen Differenzierungsdynamiken der modernen Gesellschaft moderieren, weil dieser Bezug Anschlussstellen für die Binnendiskussion schafft und gemeinsame Lernprozesse auslöst.

Allerdings bedeutet ein solcher Mediengesellschafts- und Medialisierungsbegriff auch, den bloß funktionalistisch begründeten Gegenstandsbezug des Fachs zu erweitern, sowie eine Verschiebung der Aufmerksamkeit von der Fertigung anwendungs- und berufsorientierten Wissens zur Produktion grundlagenorientierten Wissens für und 
über die Gesellschaft. Für Schritte in diese Richtung könnte die Zeit nicht besser sein: Die Reorientierungsdebatte in den Sozialwissenschaften läuft.

\section{Literatur}

Albrow, Martin (1996): The Global Age. State and Society Beyond Modernity. London: Polity Press.

Alexander, Jeffrey (1982): Theoretical Logic in Sociology. Bd. 1: Positivism, Presuppositions and Current Controversies. London: Routledge \& Kegan Paul.

Altheide, David L., Robert P. Snow (1979): Media logic. London: Sage.

Balibar, Etienne, Immanuel Wallerstein (1991): Race, Nation, Class: Ambiguous Identities. New York: Verso.

Bell, Daniel (1996 [1975]): Die nachindustrielle Gesellschaft. Frankfurt a. M.: Campus.

Bennett, W. Lance, Robert M. Entman (Hg.) (2001): Mediated Politics. Communication in the Future of Democracy. Cambridge: Cambridge University Press.

Bentele, Günter, Hans-Bernd Brosius, Otfried Jarren (Hg.) (2003): Öffentliche Kommunikation. Handbuch Kommunikations- und Medienwissenschaft. Wiesbaden: Westdeutscher Verlag.

Blum, Roger (1996): Berlusconis Modell - Parallelen in der Schweiz? In: Imhof, Kurt, Peter Schulz (Hg.): Politisches Raisonnement in der Informationsgesellschaft. Reihe: Mediensymposium Luzern. Bd. 2. Zürich: Seismo, S. 201-212.

Blumler, Jay G., Michael Gurevitch (2001): "Americanisation « reconsidered: U.K.-U.S. Campaign Communication Comparisons Across Time. In: Bennett, W. Lance, Robert M. Entman (Hg.): Mediated Politics. Communication in the Future of Democracy. Cambridge: Cambridge University Press, S. 380-403.

Boltanski, Luc, Êve Chiapello (2003 [1999]): Der neue Geist des Kapitalismus. Konstanz: UVK.

Bonfadelli, Heinz (1994): Die Wissenskluft-Perspektive. Massenmedien und gesellschaftliche Information. Konstanz: Ölschläger.

Bonfadelli, Heinz (2002): The Internet and Knowledge Gaps. A Theoretical and Empirical Investigation. In: European Journal of Communication, Nr. 17, S. 65-84.

Bourdieu, Pierre (1987): Die feinen Unterschiede. Kritik der gesellschaftlichen Urteilskraft. Frankfurt a. M.: Suhrkamp.

Bourdieu, Pierre (1998): Gegenfeuer. Wortmeldungen im Dienste des Widerstands gegen die neoliberale Invasion. Konstanz: UVK.

Donges, Patrick (2000): Amerikanisierung, Professionalisierung, Modernisierung? Anmerkungen zu einigen amorphen Begriffen. In: Kamps, Klaus (Hg.): Trans-Atlantik, Trans-Portabel? Die Amerikanisierungsthese in der politischen Kommunikation. Wiesbaden: Westdeutscher Verlag, S. $27-40$.

Donsbach, Wolfgang, Olaf Jandura (Hg.) (2003): Chancen und Gefabren der Mediendemokratie. Konstanz: UVK.

Donsbach, Wolfgang, Otfried Jarren, Hans Mathias Kepplinger, Barbara Pfetsch (1993): Beziehungsspiele - Medien und Politik in der öffentlichen Diskussion. Fallstudien und Analysen. Gütersloh: Bertelsmann Stiftung.

Eisenegger, Mark (2005): Reputation in der Mediengesellschaft. Konstitution - Issues Monitoring Issues Management. Wiesbaden: VS Verlag.

Esser, Hartmut (1988): Ethnische Diffenzierung und moderne Gesellschaft. In: Zeitschrift für Soziologie, Jg. 17, Nr. 4, S. 235-248.

Ettinger, Patrik (2005): Das Parlament in derpolitischen Kommunikation in der Schweiz. In: Donges, Patrick (Hg.): Politische Kommunikation in der Schweiz. Bern: Haupt, S. 75-90.

Featherstone, Mike (Hg.) (1990): Global Culture, Nationalism, Globalization and Modernity. London: Sage.

Gerhards, Jürgen (1993): Westeuropäische Integration und die Schwierigkeit der Entstehung einer europäischen Öffentlichkeit. In: Zeitschrift für Soziologie, Nr. 2, S. 96-110.

Gerhards, Jürgen, Friedhelm Neidhardt (1990): Strukturen und Funktionen moderner Öffentlichkeit. In: Discussion Paper, Jg. FS III. Berlin, S. 90-101. 
Gerhards, Jürgen, Jörg Rössel (1999): Zur Transnationalisierung der Gesellschaft der Bundesrepublik. Entwicklungen, Ursachen und mögliche Folgen für die europäische Integration. In: Zeitschrift für Soziologie, Jg. 28, Nr. 5, S. 325-344.

Giddens, Anthony (1976): Functionalism: Après la lutte. In: Social Research, Nr. 43, S. 325-345.

Giddens, Anthony (1991): Modernity and Self-Identity. Self and Society in the Late Modern Age. Cambridge (Mass.): Polity Press.

Giddens, Anthony (1992): Kritische Theorie der Spätmoderne. Wien: Passagen.

Habermas, Jürgen (1972): Erkenntnis und Interesse. Frankfurt a. M.: Suhrkamp.

Habermas, Jürgen (1981): Theorie des kommunikativen Handelns. 2 Bde. Frankfurt a. M.: Suhrkamp.

Habermas, Jürgen (1990 [1962]): Strukturwandel der Öffentlichkeit. Untersuchungen zu einer Kategorie der bürgerlichen Gesellschaft. Frankfurt a. M.: Suhrkamp.

Habermas, Jürgen (1992): Faktizität und Geltung. Beiträge zur Diskurstheorie des Rechts und des demokratischen Rechtsstaats. Frankfurt a. M.: Suhrkamp.

Hall, Stuart (1992): Cultural studies and its theoretical legacies. In: Großberg, L., C. Nelson, P. Treichler (Hg.), Cultural Studies. New York: Routledge, S. 277-286.

Hall, Stuart (1996 [1980]): Die zwei Paradigmen der Cultural Studies. In: Hörning, K. H., Rainer Winter (Hg.), Widerspenstige Kulturen. Cultural Studies als Herausforderung. Frankfurt a. M.: Suhrkamp, S. 13-42.

Hasebrink, Uwe (1997): Ich bin viele Zielgruppen. Anmerkungen zur Debatte um die Fragmentierung des Publikums aus kommunikationswissenschaftlicher Sicht. In: Scherer, Helmut, HansBernd Brosius (Hg.), Zielgruppen, Publikumssegmente, Nutzergruppen. Beiträge aus der Rezeptionsforschung. München: Reinhard Fischer, S. 262-280.

Heinrich, Jürgen, Frank Lobigs (2006): Publizistisches Angebot auf Lokal- und Regionalzeitungsmärkten und das Pressekartellrecht in der Schweiz und in Deutschland: Eine medienökonomische Analyse. In: Imhof, Kurt et al. (Hg.): Demokratie in der Mediengesellschaft. Reihe: Mediensymposium Luzern. Bd. 9. Wiesbaden: VS Verlag.

Heitmeyer, Wilhelm, Joachim Müller, Helmut Schröder (1997): Verlockender Fundamentalismus. Türkische Jugendliche in Deutschland. Frankfurt a. M.: Suhrkamp.

Hoffmann-Nowotny, Hans-Joachim (2001): Das Fremde in der Schweiz. Ergebnisse soziologischer Forschung. Zürich: Seismo.

Holtz-Bacha, Christina (Hg.) (1999): Wablkampf in den Medien - Wablkampf mit den Medien. Ein Reader zum Wabljabr 1998. Wiesbaden: Westdeutscher Verlag.

Hondrich, Karl Otto (2002): Enthüllung und Entrüstung. Eine Phänomenologie des politischen Skandals. Frankfurt a. M.: Suhrkamp.

Horkheimer, Max, Theodor Adorno (1969 [1944/1947]): Dialektik der Aufklärung. Philosophische Fragmente. Frankfurt a. M.: Fischer.

Imhof, Kurt (1996): Eine Symbiose: Soziale Bewegungen und Medien. In: Imhof, Kurt, Peter Schulz (Hg.): Politisches Raisonnement in der Informationsgesellschaft. Reihe: Mediensymposium Luzern. Bd. 2. Zürich: Seismo, S. 165-186.

Imhof, Kurt (1997): Gemeinschaft und Gesellschaft: Minderheitensoziologie und soziale Integration. In: Hradil, Stefan (Hg.): Differenz und Integration. Die Zukunft moderner Gesellschaften. Verhandlungen des 28. Kongresses der Deutschen Gesellschaft für Soziologie 1996 in Dresden. Frankfurt a. M.: Campus, S. 861-875.

Imhof, Kurt (1999): Die Privatisierung des Öffentlichen: Zum Siegeszug der Primärgruppenkommunikation in den Medien. In: Honegger, Claudia, Stefan Hradil, Franz Traxler (Hg.): Grenzenlose Gesellschaft? Verhandlungen des 29. Kongresses der Deutschen Gesellschaft für Soziologie 1998 in Freiburg i. Br. Opladen: Leske + Budrich, S. 717-732.

Imhof, Kurt (2000): Öffentlichkeit und Skandal. In: Neumann-Braun, Klaus, Stefan MüllerDoohm (Hg.): Medien- und Kommunikationssoziologie: Eine Einführung in zentrale Begriffe und Theorien. Weinheim: Juventa, S. 55-68.

Imhof, Kurt (2002): Öffentlichkeit und Identität. In: Kaelble, Hartmut, Martin Kirsch, Alexander Schmidt-Gernig (Hg.): Transnationale Öffentlichkeiten und Identitäten im 20. Jabrbundert. Frankfurt a. M.: Campus, S. 37-56. 
Imhof, Kurt (2003): Wandel der Gesellschaft im Licht öffentlicher Kommunikation. In: Behmer, Markus et al. (Hg.): Medienentwicklung und gesellschaftlicher Wandel. Beiträge zu einer theoretischen und empirischen Herausforderung und Geschichte. Wiesbaden: Westdeutscher Verlag, S. 153-182.

Imhof, Kurt (2006 [1996]): Diskontinuität der Moderne. Theorie des sozialen Wandels. Reihe «Theorie und Gesellschaft», Frankfurt a. M: Campus.

Imhof, Kurt, Thomas Eberle (Hg.) (2005): Triumph und Elend des Neoliberalismus. Zürich: Seismo.

Imhof, Kurt, Mark Eisenegger (1999): Inszenierte Politik: Resonanz von "Events in den Medien. In: Szyszka, Peter (Hg.): Öffentlichkeit. Diskurs zu einem Schlüsselbegriff der Organisationskommunikation. Wiesbaden: Westdeutscher Verlag, S. 195-218.

Imhof, Kurt, Otfried Jarren, Roger Blum (Hg.) (1999): Stenerungs- und Regelungsprobleme in der Informationsgesellschaft. Reihe: Mediensymposium Luzern. Bd. 5. Wiesbaden: Westdeutscher Verlag.

Imhof, Kurt, Otfried Jarren, Roger Blum (Hg.) (2002): Integration und Medien. Reihe: Mediensymposium Luzern. Bd. 7. Wiesbaden: Westdeutscher Verlag.

Imhof, Kurt, Esther Kamber (2001): Politik und Gesellschaft im Strukturwandel der Öffentlichkeit. Zur Veränderung der Konstitutionslogiken der öffentlichen Kommunikation. In: Allmendiger, Jutta (Hg.): Gute Gesellschaft? Verhandlungen des 30. Kongresses der Deutschen Gesellschaft für Soziologie in Köln 2000. Opladen: Leske + Budrich, S. 425-453.

Imhof, Kurt, Heinz Kleger, Gaetano Romano (Hg.) (1996): Konkordanz und Kalter Krieg. Analyse von Medienereignissen in der Schweiz der Zwischen- und Nachkriegszeit. Reihe: Krise und sozialer Wandel. Bd. 2. Zürich: Seismo.

Imhof, Kurt, Heinz Kleger, Gaetano Romano (Hg.) (1999): Vom kalten Krieg zur Kulturrevolution. Analyse von Medienereignissen in der Schweiz der 50 er und 60er Jabre. Reihe: Krise und sozialer Wandel. Bd. 3. Zürich: Seismo.

Imhof, Kurt, Peter Schulz (Hg.) (1996): Politisches Raisonnement in der Informationsgesellschaft. Reihe: Mediensymposium Luzern. Bd. 2. Zürich: Seismo.

Imhof, Kurt, Roger Blum, Heinz Bonfadelli, Otfried Jarren (Hg.) (2004): Mediengesellschaft: Strukturen, Merkmale, Entwicklungsdynamiken. Reihe: Mediensymposium Luzern. Bd. 8. Wiesbaden: VS Verlag.

Imhof, Kurt, Roger Blum, Heinz Bonfadelli, Otfried Jarren (Hg.) (2006): Demokratie in der Mediengesellschaft. Reihe: Mediensymposium Luzern. Bd. 9. Wiesbaden: VS Verlag.

Jarren, Otfried (1994): Mediengewinne und Institutionenverluste? - Zum Wandel des intermediären Systems in der Mediengesellschaft. Theoretische Anmerkungen zum Bedeutungszuwachs elektronischer Medien in der politischen Kommunikation. In: Jarren, Otfried, Uwe Hasebrink (Hg.): Politische Kommunikation in Hörfunk und Fernseben. Opladen: Leske + Budrich, S. 23-34.

Jarren, Otfried (1996): Auf dem Weg in die "Mediengesellschaft «? Medien als Akteure und institutionalisierter Handlungskontext. Theoretische Anmerkungen zum Wandel des intermediären Systems. In: Imhof, Kurt, Peter Schulz (Hg.): Politisches Raisonnement in der Informationsgesellschaft. Reihe: Mediensymposium Luzern. Bd. 2. Zürich: Seismo, S. 79-96.

Jarren, Otfried (1998): Medien, Mediensystem und politische Öffentlichkeit im Wandel. In: Sarcinelli, Ulrich (Hg.): Politikvermittlung und Demokratie in der Mediengesellschaft. Beiträge zur politischen Kommunikationskultur. Wiesbaden: Westdeutscher Verlag, S. 74-96.

Jarren, Otfried (2001): Mediengesellschaft-Risiken für die politische Kommunikation. In: Beilage zur Wochenzeitung »Das Parlament«, Jg. 41-42, S. 10-19.

Jarren, Otfried, Kurt Imhof, Roger Blum (Hg.) (2000): Zerfall der Öffentlichkeit? Reihe: Mediensymposium Luzern. Bd. 6. Wiesbaden: Westdeutscher Verlag.

Jarren, Otfried, Ulrich Sarcinelli, Ulrich Saxer (Hg.) (1998): Politische Kommunikation in der demokratischen Gesellschaft. Wiesbaden: Westdeutscher Verlag.

Jarren, Otfried, Heribert Schatz, Hartmut Weßler (Hg.) (1996): Medien und politischer Prozeß. Politische Öffentlichkeit und massenmediale Politikvermittlung im Wandel. Wiesbaden: Westdeutscher Verlag. 
Joas, Hans (1992): Die Kreativität des Handelns. Frankfurt a. M.: Suhrkamp.

Joas, Hans (1996): Die Modernität des Krieges. In: Leviathan, Nr. 1, S. 13-27.

Kaase, Max (1998): Demokratisches System und die Mediatisierung von Politik. In: Sarcinelli, Ulrich (Hg.): Politikvermittlung und Demokratie in der Mediengesellschaft Beiträge zur politischen Kommunikationskultur. Wiesbaden: Westdeutscher Verlag, S. 24-51.

Kaelble, Hartmut, Martin Kirsch, Alexander Schmidt-Gernig (Hg.) (2002): Transnationale Öffentlichkeiten und Identitäten im 20. Jabrhundert. Frankfurt a. M.: Campus.

Kamber, Esther, Kurt Imhof (2004): Phänomenologie der politischen Kommunikation. In: Medienwissenschaft Schweiz, Nr. 1, S. 10-18.

Kamber, Esther, Mario Schranz (2002): Von der Herstellung zur Darstellung demokratischer Öffentlichkeit. Die Politikvermittlung der Massenmedien unter den Bedingungen eines ausdifferenzierten Mediensystems. In: Imhof, Kurt, Otfried Jarren, Roger Blum (Hg.): Integration und Medien. Reihe: Mediensymposium Luzern. Bd. 7.Wiesbaden: Westdeutscher Verlag, S. 347363.

Kepplinger, Hans Mathias (1992): Ereignismanagement. Wirklichkeit und Massenmedien. Zürich: Edition Interfrom.

Kepplinger, Hans Mathias (1996): Skandale und Politikverdrossenheit - ein Langzeitvergleich. In: Jarren, Otfried, Heribert Schatz, Hartmut Weßler (Hg.): Medien und politischer Prozess. Politische Öffentlichkeit und massenmediale Politikvermittlung im Wandel. Wiesbaden: Westdeutscher Verlag, S. 41-58.

Kepplinger, Hans Mathias (2002): Mediatization of Politics: Theory and Data. In: Journal of Communication, Jg. 52, Nr. 4, S. 972-986.

Knoche, Manfred (1997): Medienkonzentration und publizistische Vielfalt. Legitimationsgrenzen des privatwirtschaftlichen Mediensystems. In: Renger, Rudi, Gabriele Siegert (Hg.): Kommunikationswelten. Wissenschaftliche Perspektiven zur Medien-und Informationsgesellschaft. Wien: Studienverlag, S. 123-158.

Knorr-Cetina, Karin (1999): Epistemic cultures. How the sciences make knowledge. Cambridge (Mass.): Harvard University Press.

Krotz, Friedrich (2002): Die Mediatisierung von Alltag und sozialen Beziebungen und die Formen sozialer Integration. In: Imhof, Kurt, Otfried Jarren, Roger Blum (Hg.): Integration und Medien. Wiesbaden: Westdeutscher Verlag, Bd. 7, S. 168-183.

Lash, Scott, John Urry (1994): Economies of signs and space. London: Sage.

Leggewie, Claus, Sigrid Baringhorst (1990): Multi Kulti. Spielregeln für die Vielvölkerpolitik. Berlin: Rotbuch.

Lockwood, David (1964): Social Integration and System Integration. In: Zollschan, George K., Walter Hirsch (eds.): Explorations in Social Change. New York: Houghton-Mifflin, S. 244257.

Luhmann, Niklas (1984): Soziale Systeme. Grundriß einer allgemeinen Theorie. Frankfurt a. M.: Suhrkamp.

Luhmann, Niklas (1990): Gesellschaftliche Komplexität und öffentliche Meinung. In: Luhmann, Niklas: Soziologische Aufklärung. Wiesbaden: Westdeutscher Verlag, Bd. 5, S. 170-182.

Luhmann, Niklas (1996): Die Realität der Massenmedien. Wiesbaden: Westdeutscher Verlag.

Marcinkowski, Frank (1993): Publizistik als autopoietisches System. Wiesbaden: Westdeutscher Verlag.

Marcinkowski, Frank (2000): Die Medien-Öffentlichkeit des Parlaments in der Verhandlungsdemokratie. In: Jarren, Otfried, Kurt Imhof, Roger Blum (Hg.): Zerfall der Öffentlichkeit? Reihe: Mediensymposium Luzern. Bd. 6. Wiesbaden: Westdeutscher Verlag, S. 49-73.

Marr, Mirko (2004): Wer hat Angst vor der digitalen Spaltung? Zur Haltbarkeit des Bedrohungsszenarios. In: Medien \& Kommunikationswissenschaft, Jg. 52, Nr. 1, S. 76-94.

McQuail, Denis (2000): McQuail's Mass Communication Theory. London.

Meier, Werner A. (2004): Informationsgesellschaft oder Mediengesellschaft? In: Imhof, Kurt et al. (Hg.): Mediengesellschaft: Strukturen, Merkmale, Entwicklungsdynamiken. Reihe: Mediensymposium Luzern. Bd. 8. Wiesbaden: VS Verlag, S. 57-78.

Meier, Werner A., Josef Trappel (2005): Die transnationale Vermachtung durch Medienkonzerne 
als Voraussetzung für Europäische Öffentlichkeit? In: Langenbucher, Wolfgang R., Michael Latzer (Hg.): Medialer Wandel und Europäische Öffentlichkeit: Eine transnationale Perspektive. Wiesbaden: VS Verlag, S. 262-275.

Meier-Braun, Karl-Heinz, Martin A. Kilgus (Hg.) (2002): Integration durch Politik und Medien? 7. Medienforum Migranten bei uns. Baden-Baden: Nomos.

Müller-Doohm, Stefan (1998): Medienkultur und Globalität. In: Imhof, Kurt, Peter Schulz (Hg.): Die Veröffentlichung des Privaten - die Privatisierung des Öffentlichen. Reihe: Mediensymposium Luzern. Bd. 4.Wiesbaden: Westdeutscher Verlag, S. 471-486.

Münch, Richard (1995): Dynamik der Kommunikationsgesellschaft. Frankfurt a. M.: Suhrkamp.

Münch, Richard (1997): Mediale Ereignisproduktion: Strukturwandel der politischen Macht. In: Hradil, Stefan (Hg.): Differenz und Integration. Die Zukunft moderner Gesellschaften. Verhandlungen des 28. Kongresses der Deutschen Gesellschaft für Soziologie 1996 in Dresden. Frankfurt a. M.: Campus, S. 696-709.

Nassehi, Armin (1990): Zum Funktionswandel der Ethnizität im Prozess gesellschaftlicher Modernisierung. Ein Beitrag zur Theorie der funktionalen Differenzierung. In: Soziale Welt, Jg. 3, S. 261-282.

Neckel, Sighard (1986): Das Stellhölzchen der Macht. Zur Soziologie des politischen Skandals. In: Leviathan, Jg. 14, Nr. 4, S. 581-605.

Negrine, Ralph (1998): Parliament and the media. A study of Britain, Germany and France. London: Pinter

Negt, Oskar, Alexander Kluge (1972): Öffentlichkeit und Erfahrung. Zur Organisationsanalyse von bürgerlicher und proletarischer Öffentlichkeit. Frankfurt a. M.: Suhrkamp.

Neidhardt, Friedhelm (2005): Europäische Öffentlichkeit als Prozess. Anmerkungen zum Forschungsstand. In: Langenbucher, Wolfgang R., Michael Latzer (Hg.): Medialer Wandel und Europäische Öffentlichkeit: Eine transnationale Perspektive. Wiesbaden: VS Verlag, S. 46-61.

Neidhardt, Friedhelm (Hg.) (1994): Öffentlichkeit, öffentliche Meinung, soziale Bewegungen. Sonderheft 34 der KZfSS. Wiesbaden: Westdeutscher Verlag.

Neidhardt, Friedhelm, Dieter Rucht (1993): Auf dem Weg in die Bewegungsgesellschaft? Über die Stabilisierbarkeit sozialer Bewegungen. In: Soziale Welt, Nr. 3, S. 305-326.

Norris, Pippa (2001): Digital divide. Civic engagement, information poverty, and the Internet worldwide. Cambridge: Cambridge University Press.

Parsons, Talcott (1976 [1951]): Der Begriff der Gesellschaft: Seine Elemente und ibre Verknüpfungen. In: Jensen, Stefan (Hg.): Zur Theorie sozialer Systeme. Wiesbaden: Westdeutscher Verlag, Bd. 14, S. 121-160.

Peters, Bernhard (1993a): Die Integration moderner Gesellschaften. Frankfurt a. M.: Suhrkamp.

Peters, Bernhard (1993b): Die Verselbständigung des Sozialen. In: Peters, Bernhard: Die Integration moderner Gesellschaften. Frankfurt a. M.: Suhrkamp, S. 229-303.

Peters, Birgit (1994): Öffentlichkeitselite - Bedingung und Bedeutung von Prominenz. In: Neidhardt, Friedhelm (Hg.): Öffentlichkeit, öffentliche Meinung, soziale Bewegungen. Sonderheft 34 der KZfSS. Wiesbaden: Westdeutscher Verlag, S. 191-213.

Pfetsch, Barbara (1998): Bürger - Publikum. In: Jarren, Otfried, Ulrich Sarcinelli, Ulrich Saxer (Hg.): Politische Kommunikation in der demokratischen Gesellschaft. Wiesbaden: Westdeutscher Verlag, S. 406-413.

Picard, Robert, G. (2001): Relations among Media Economics, Content and Diversity. In: Nordicom Review 22, Heft 1, S. 65-69.

Pongs, Armin (1999/2000): In welcher Gesellschaft leben wir eigentlich? Gesellschaftskonzepte im Vergleich. Bd. 1 (1999), Bd. 2 (2000). München: Dilemma Verlag.

Postman, Neil (1985): Amusing Ourselves to Death. New York: Viking-Penguin.

Robertson, Roland (1992): Globalization. Social Theory and Global Culture. London.

Rucht, Dieter (1994): Öffentlichkeit als Mobilisierungsfaktor für soziale Bewegungen. In: Neidhardt, Friedhelm (Hg.): Öffentlichkeit, öffentliche Meinung, soziale Bewegungen. Sonderheft 34 der KZfSS. Wiesbaden: Westdeutscher Verlag, S. 337ff.

Rucht, Dieter (2003): Zum Wandel politischen Protests in der Bundesrepublik. Verbreiterung, Professionalisierung, Trivialisierung. In: Vorgänge, Jg. 4, Nr. 03, S. 4-11. 
Sarcinelli, Ulrich (1987): Symbolische Politik zur Bedeutung symbolischen Handelns in der Wablkampfkommunikation der Bundesrepublik Deutschland. Wiesbaden: Westdeutscher Verlag.

Sarcinelli, Ulrich (1994): Mediale Politikdarstellung und politisches Handeln: analytische Anmerkungen zu einer notwendigerweise spannungsreichen Beziehung. In: Jarren, Otfried ( $\mathrm{Hg}$.): Politische Kommunikation in Hörfunk und Fernsehen. Opladen: Leske + Budrich, S. 35-50.

Sarcinelli, Ulrich (Hg.) (1998): Politikvermittlung und Demokratie in der Mediengesellschaft. Beiträge zur politischen Kommunikationskultur. Wiesbaden: Westdeutscher Verlag.

Sarcinelli, Ulrich, Martin Wissel (1998): Mediale Politikvermittlung, politische Beteiligung und politische Bildung: Medienkompetenz als Basisqualifikation in der demokratischen Bürgergesellschaft. In: Sarcinelli, Ulrich (Hg.): Politikvermittlung und Demokratie in der Mediengesellschaft. Beiträge zur politischen Kommunikationskultur. Wiesbaden: Westdeutscher Verlag, S. 408-427.

Saxer, Ulrich (1998): System, Systemwandel und politische Kommunikation. In: Jarren, Otfried, Ulrich Sarcinelli, Ulrich Saxer (Hg.): Politische Kommunikation in der demokratischen Gesellschaft. Wiesbaden: Westdeutscher Verlag, S. 21-63.

Saxer, Ulrich (2004): Mediengesellschaft: Auf dem Weg zu einem Konzept. In: Imhof, Kurt et al. (Hg.): Mediengesellschaft: Strukturen, Merkmale, Entwicklungsdynamiken. Reihe: Mediensymposium Luzern. Bd. 8.Wiesbaden: VS Verlag, S. 159-174.

Schenk, Michael (1998): Mediennutzung und Medienwirkung als sozialer Prozess. In: Sarcinelli, Ulrich (Hg.): Politikvermittlung und Demokratie in der Mediengesellschaft. Beiträge zur politischen Kommunikationskultur. Wiesbaden: Westdeutscher Verlag, S. 387-407.

Schmitt-Beck, Rüdiger, Barbara Pfetsch (1994): Politische Akteure und die Medien der Massenkommunikation. Zur Generierung von Öffentlichkeit in Wablkämpfen. In: Neidhardt, Friedhelm (Hg.): Öffentlichkeit, öffentliche Meinung, soziale Bewegungen. Sonderheft 34 der KZfSS. Wiesbaden: Westdeutscher Verlag, S. 106-138.

Schmolke, Michael (1996): Von der Parteipresse zur Medienpartei: ein fragwürdiges Verbältnis unter dem Druck der real existierenden Politik. In: Imhof, Kurt, Peter Schulz (Hg.): Politisches Raisonnement in der Informationsgesellschaft. Reihe: Mediensymposium Luzern. Bd. 2. Zürich: Seismo, S. 187-200.

Schranz, Mario (2000): Die Debatte über die Asyl- und Flüchtlingsproblematik in der öffentlichen politischen Kommunikation der Schweiz. Zur Thematisierung des "Fremden " im Fernsehen DRS und zentralen Printmedien der deutschen Schweiz von 1985-1996. Zürich: Lizentiatsarbeit an der Phil. Fakultät I der Universität Zürich.

Schulz, Winfried (2004): Reconstructing Mediatization as an Analytical Concept. In: European Journal of Communication, Jg. 19, Nr. 1, S. 87-101.

Schulze, Gerhard (1993): Die Erlebnisgesellschaft. Kultursoziologie der Gegenwart. Frankfurt a. M.: Campus.

Schwengel, Hermann (1997): Vor und nach dem Medienspiel. Globalisierung als kulturelle Verfassung der Moderne. In: Hradil, Stefan (Hg.): Differenz und Integration. Die Zukunft moderner Gesellschaften. Verhandlungen des 28. Kongresses der Deutschen Gesellschaft für Soziologie 1996 in Dresden. Frankfurt a. M.: Campus, S. 663-678.

Siegert, Gabriele (1998): „Guten Abend, liebe Zielgruppen“. Das entsubjektivierte Publikum als Markt und „coin of exchange“. In: Medien Journal 22, Heft 4, S. 48-58.

Siegert, Gabriele (2003): Im Zentrum des Taifuns: Die Ökonomisierung als treibende Kraft des medialen Wandels. In: Medien Journal 27, Heft 1, S. 20-30.

Siegert, Gabriele, Werner A. Meier, Josef Trappel (2005): Auswirkungen der Ökonomisierung auf Medien und Inhalte. In: Heinz Bonfadelli, Otfried Jarren, Gabriele Siegert (Hg.): Einführung in die Publizistikwissenschaft. Bern: Haupt, S. 469-494.

Theis-Berglmair/Bentele bzw. Selbstverständnisausschuss der DGPuK (2001): Die Mediengesellschaft und ibre Wissenschaft. Herausforderungen für die Kommunikations- und Medienwissenschaft als akademische Disziplin. Selbstverständnispapier der Deutschen Gesellschaft für Publizistik-und Kommunikationswissenschaft (DGPuK) vom Januar 2001.

Tobler, Stefan (2004): Aufstieg und Fall der New Economy. In: Imhof, Kurt et al. (Hg.): Mediengesellschaft: Strukturen, Merkmale, Entwicklungsdynamiken. Reihe: Mediensymposium Luzern. Bd. 8. Wiesbaden: VS Verlag, S. 231-261. 
Tönnies, Ferdinand (1922): Kritik der öffentlichen Meinung. Berlin: Springer.

Trappel, Josef, Werner A. Meier, Klaus Schrape, Michaela Wölk (Hg.) (2002): Die gesellschaftlichen Folgen der Medienkonzentration. Veränderungen in den demokratischen und kulturellen Grundlagen der Gesellschaft. Wiesbaden: Westdeutscher Verlag.

Viswanath, Kasisomayajula, John R. Finnegan (1996): The Knowledge Gap Hypothesis. In: Communication Yearbook, Nr. 19, S. 187-227.

Wallerstein, Immanuel (1979): The Capitalist World-Economy. Essays. Cambridge: Cambridge University Press.

Wehler, Hans-Ulrich (1975): Modernisierungstheorie und Geschichte. Göttingen: Vandenhoeck \& Ruprecht.

Welsch, Wolfgang (1995): Vernunft. Die zeitgenössische Vernunftkritik und das Konzept der transversalen Vernunft. Frankfurt a. M.: Suhrkamp.

Wilke, Jürgen (1996): Status und Medienprominenz. In: Imhof, Kurt, Peter Schulz (Hg.): Politisches Raisonnement in der Informationsgesellschaft. Reihe: Mediensymposium Luzern. Bd. 2. Zürich: Seismo, S. 99-106.

Willke, Helmut (1992): Ironie des Staates. Grundlinien einer Staatstheorie polyzentrischer Gesellschaft. Frankfurt a. M.: Suhrkamp.

Wimmer, Andreas (1996): Der Appell an die Nation. Kritische Bemerkungen zu vier Analysen von Xenophobie und Rassismus. In: Wicker, Hans-Rudolf et al. (Hg.): Das Fremde in der Gesellschaft. Migration, Ethnizität und Staat. Zürich: Seismo, S. 173-198.

Winter, Carsten, Tanja Thomas, Andreas Hepp (Hg.) (2003): Medienidentitäten. Identitäten im Kontext von Globalisierung und Medienkultur. Köln: Von Halem.

Winter, Rainer (2001): Die Kunst des Eigensinns. Cultural Studies als Kritik der Macht. Weilerswist: Velbrück.

Wirth, Werner (1997): Von der Information zum Wissen. Die Rolle der Rezeption für die Entstebung von Wissensunterschieden. Ein Beitrag zur Wissenskluftforschung. Wiesbaden: Westdeutscher Verlag. 